\title{
The effect of classical noise on a quantum two-level system
}

\author{
Jean-Philippe Aguilar, Nils Berglund
}

\begin{abstract}
We consider a quantum two-level system perturbed by classical noise. The noise is implemented as a stationary diffusion process in the off-diagonal matrix elements of the Hamiltonian, representing a transverse magnetic field. We determine the invariant measure of the system and prove its uniqueness. In the case of Ornstein-Uhlenbeck noise, we determine the speed of convergence to the invariant measure. Finally, we determine an approximate one-dimensional diffusion equation for the transition probabilities. The proofs use both spectral-theoretic and probabilistic methods.
\end{abstract}

Date. May 7, 2008.

2000 Mathematical Subject Classification. 60h10, 35P15 (primary), 81Q15, 93E03 (secondary)

Keywords and phrases. spin 1/2, noise, heat bath, open systems, stochastic differential equations, Ornstein-Uhlenbeck process, transition times, transition probabilities, spectral gap, diffusions on Lie groups, Stroock-Varadhan theorem, averaging, renewal equations, Laplace transforms

\section{Introduction}

Noise is often used as a model for the effect of the environment (for instance a heat bath) on a relatively small system. In general, it is difficult to prove rigorously from first principles that a stochastic model indeed gives a good approximation of the real dynamics. However, such a proof has been obtained in a number of specific classical systems, see for instance [FKM65, SL77, EPRB99, RBT00, RBT02].

For quantum systems, the theory of effective stochastic models is not yet so welldeveloped. One approach in which progress has been made in recent years is the approach of repeated quantum interactions. If the quantum system is assumed to interact during successive short time intervals with independent copies of a quantum heat reservoir, one can under certain assumptions derive effective equations involving quantum noises. See for instance [AP06, BJM06, AJ07a, AJ07b] for recent works in this direction.

In the present work, we consider the intermediate situation of a classical noise acting on a quantum system. We shall focus on the simplest possible quantum system, namely a spin-1/2. The classical noise is realised by adding off-diagonal stochastic processes in the system's Hamiltonian. This situation can be realised, for instance, by letting the spin interact with a magnetic field subject to small stochastic fluctuations, due to the magnet creating the field being subject to weak random noise.

When the spin is prepared, say, in the "down" state, and subjected to a constant magnetic field in the $z$-direction, it will remain in the same state for ever. If the direction of the magnetic field is allowed to fluctuate in time, however, transitions to the "spin-up" state become possible. The aim of this work is to estimate how long it takes for these transitions to occur, depending on the characteristics of the noise. 
This paper is organised as follows. Section 2 contains a detailed description of the model and all results. The main results are the following: Theorem 2.3 shows that under rather general assumptions on the noise, the system admits a unique invariant measure induced by the Haar measure on SU(2). Theorem 2.6 gives a spectral-gap estimate in the case of Ornstein-Uhlenbeck noise, describing the speed of convergence to the invariant measure, as a function of noise and coupling intensity, and of the rate of decay of correlations of the noise term. Finally, Theorem 2.7 uses an effective one-dimensional diffusion approximation for the transition probability to derive expected transition times. Sections 3, 4 and 5] contain the proofs of these results.

\section{$2 \quad$ Model and results}

\subsection{Definition of the model}

The simplest possible quantum system is a two-level system (e.g. a spin 1/2), which can be described by the unperturbed Hamiltonian

$$
H_{0}=\left(\begin{array}{cc}
\frac{1}{2} & 0 \\
0 & -\frac{1}{2}
\end{array}\right)
$$

acting on the Hilbert space $\mathcal{H}=\mathbb{C}^{2}$. We would like to perturb this system by "classical noise", able to induce transitions between the two energy levels. The noise should however preserve the unitary character of the quantum evolution. A way to do this is to add a non-diagonal interaction term to the Hamiltonian, of the form $\kappa V(t)$, with

$$
V(t)=\left(\begin{array}{cc}
0 & Z_{t} \\
Z_{t} & 0
\end{array}\right), \quad Z_{t}=X_{t}+\mathrm{i} Y_{t}
$$

where $\left\{Z_{t}\right\}_{t \geqslant 0}$ is an ergodic, stationary Markov process on some filtered probability space. This is equivalent to assuming that the spin $1 / 2$ is interacting with a magnetic field $B_{t}=\left(2 \kappa X_{t}, 2 \kappa Y_{t}, 1\right)$, since the total Hamiltonian can be written

$$
H(t)=H_{0}+\kappa V(t)=\kappa X_{t} \sigma^{1}+\kappa Y_{t} \sigma^{2}+\frac{1}{2} \sigma^{3}=: \frac{1}{2} B_{t} \cdot \sigma,
$$

where the $\sigma^{i}$ are the Pauli matrices. For definiteness, we shall assume that $Z_{t}$ is the solution of an Itô stochastic differential equation (SDE) of the form

$$
\mathrm{d} Z_{t}=f\left(Z_{t}\right) \mathrm{d} t+g\left(Z_{t}\right) \mathrm{d} W_{t}
$$

where $\left\{W_{t}\right\}_{t \geqslant 0}$ denotes a standard Brownian motion, and $f$ and $g$ satisfy the usual Lipshitz and bounded-growth conditions ensuring existence of a pathwise unique strong solution for any initial condition. A typical choice would be an Ornstein-Uhlenbeck process, defined by the SDE

$$
\mathrm{d} Z_{t}=-\gamma Z_{t} \mathrm{~d} t+\sigma \mathrm{d} W_{t},
$$

where the initial condition $Z_{0}$ is a centred Gaussian of variance $\sigma^{2} / 2 \gamma$. This represents the situation of the magnet generating the field being subjected to a harmonic potential and white noise. This choice is also motivated by the fact that in certain situations, the effect of a classical heat bath on a small (classical) system has been rigorously shown to be describable by such an Ornstein-Uhlenbeck process [EPRB99]. One can, however, consider 
other types of stochastic processes as well, and part of our results do not depend on the detailed definition of $Z_{t}$. Furthermore, one can easily deal with more general interaction terms than (2.5), including several sources of noise for instance. However, the situation with a single noise is in some sense the most interesting one, since it is in this situation that noise-induced transitions are the most difficult.

The evolution of the coupled system is governed by the time-dependent Schrödinger equation

$$
\mathrm{id} \psi_{t}=H(t) \psi_{t} \mathrm{~d} t .
$$

Provided the stochastic process $Z_{t}$ has continuous sample paths, which are stochastically bounded on compact time intervals 1 (as is the case for the Ornstein-Uhlenbeck process), Dyson's theorem implies the existence of a unique two-parameter family of unitary operators $U(t, s) \in \mathrm{U}(2)$, forming a (strongly) continuous semi-group and such that

$$
\psi_{t}=U(t, s) \psi_{s}
$$

holds almost surely for all $t>s$. In our case, $H(t)$ having zero trace, we have in fact $U(t, s) \in \mathrm{SU}(2)$. For fixed $s$, the process $t \mapsto U_{t}=U(t, s)$ satisfies the equation

$$
\operatorname{id} U_{t}=H(t) U_{t} \mathrm{~d} t .
$$

Thus, characterising the evolution of the quantum system is equivalent to characterising the stochastic process $\left\{U_{t}\right\}_{t \geqslant 0}$ on $\mathrm{SU}(2)$.

\subsection{Coordinates on $\mathrm{SU}(2)$}

In order to study the solutions of Equation (2.8), we have to choose a convenient parametrisation of the Lie group $\mathrm{SU}(2)$. A possible choice is to decompose elements of $\mathrm{SU}(2)$ on a basis of identity and Pauli matrices as

$$
U=\mathrm{i}\left(x_{1} \sigma^{1}+x_{2} \sigma^{2}+x_{3} \sigma^{3}\right)+x_{4} \mathbb{1}=\left(\begin{array}{rr}
x_{4}+\mathrm{i} x_{3} & x_{2}+\mathrm{i} x_{1} \\
-x_{2}+\mathrm{i} x_{1} & x_{4}-\mathrm{i} x_{3}
\end{array}\right),
$$

where the $x_{i}$ are real and satisfy

$$
x_{1}^{2}+x_{2}^{2}+x_{3}^{2}+x_{4}^{2}=1 .
$$

Plugging into (2.8) and using Itô's formula, one gets the system of equations

$$
\begin{aligned}
\mathrm{d} x_{1, t} & =\left[-\frac{1}{2} x_{2, t}-\kappa X_{t} x_{4, t}-\kappa Y_{t} x_{3, t}\right] \mathrm{d} t, \\
\mathrm{~d} x_{2, t} & =\left[\frac{1}{2} x_{1, t}-\kappa X_{t} x_{3, t}+\kappa Y_{t} x_{4, t}\right] \mathrm{d} t, \\
\mathrm{~d} x_{3, t} & =\left[-\frac{1}{2} x_{4, t}+\kappa X_{t} x_{2, t}+\kappa Y_{t} x_{1, t}\right] \mathrm{d} t, \\
\mathrm{~d} x_{4, t} & =\left[\frac{1}{2} x_{3, t}+\kappa X_{t} x_{1, t}-\kappa Y_{t} x_{2, t}\right] \mathrm{d} t .
\end{aligned}
$$

However, one of these equations is redundant because of Condition (2.10). It is preferable to work with a three-dimensional parametrisation of $\mathrm{SU}(2)$. A classical way to do this is to write $U \in \mathrm{SU}(2)$ as the exponential $U=\mathrm{e}^{\mathrm{i} M}$, where $M$ lives in the Lie algebra $\mathfrak{s u}(2)$. Decomposing $M$ on the basis of Pauli matrices as $M=r\left(\hat{y}_{1} \sigma^{1}+\hat{y}_{2} \sigma^{2}+\hat{y}_{3} \sigma^{3}\right)$, with $\hat{y}_{1}^{2}+\hat{y}_{2}^{2}+\hat{y}_{3}^{2}=1$, yields the representation

$$
U=\left(\begin{array}{cc}
\cos r+\mathrm{i} \hat{y}_{3} \sin r & {\left[\mathrm{i} \hat{y}_{1}+\hat{y}_{2}\right] \sin r} \\
{\left[\mathrm{i} \hat{y}_{1}-\hat{y}_{2}\right] \sin r} & \cos r-\mathrm{i} \hat{y}_{3} \sin r
\end{array}\right)
$$

\footnotetext{
${ }^{1}$ In other words, $\lim _{L \rightarrow \infty} \mathbb{P}\left\{\sup _{0 \leqslant t \leqslant T}\left|Z_{t}\right|>L\right\}=0$ for all $T>0$.
} 
One can then write the $\hat{y}_{i}$ in spherical coordinates in order to obtain a three-dimensional system equivalent to (2.8). However, the resulting system turns out to have a rather complicated form.

After some trials, one finds that the best suited parametrisation of $\mathrm{SU}(2)$ for the present situation is given by

$$
U=u(\chi, \phi, \psi):=\left(\begin{array}{cc}
\cos \chi \mathrm{e}^{-\mathrm{i}(\phi / 2+\psi)} & \sin \chi \mathrm{e}^{-\mathrm{i}(\phi / 2-\psi)} \\
-\sin \chi \mathrm{e}^{\mathrm{i}(\phi / 2-\psi)} & \cos \chi \mathrm{e}^{\mathrm{i}(\phi / 2+\psi)}
\end{array}\right)
$$

The variable $\chi$ lives in the interval $[0, \pi / 2]$, while the pair $(\phi / 2+\psi, \phi / 2-\psi)$ lives in the two-torus $\mathbb{T}^{2} 2$

Proposition 2.1. The system (2.8) is equivalent to the system

$$
\begin{aligned}
\mathrm{d} \chi_{t} & =\kappa\left[X_{t} \sin \phi_{t}+Y_{t} \cos \phi_{t}\right] \mathrm{d} t, \\
\mathrm{~d} \phi_{t} & =\left(1+\frac{2 \kappa}{\tan 2 \chi_{t}}\left[X_{t} \cos \phi_{t}-Y_{t} \sin \phi_{t}\right]\right) \mathrm{d} t, \\
\mathrm{~d} \psi_{t} & =-\frac{\kappa}{\sin 2 \chi_{t}}\left[X_{t} \cos \phi_{t}-Y_{t} \sin \phi_{t}\right] \mathrm{d} t .
\end{aligned}
$$

PROOF: This is a straightforward application of Itô's formula. The second-order term in the formula actually vanishes, due to the fact that the diffusion term only enters indirectly, via the equation defining $Z_{t}$, and the change of variables is independent of $Z$.

Notice that the system (2.14) has a skew-product structure, as the right-hand side does not depend on $\psi_{t}$. Thus in fact all the important information on the dynamics is contained in the first two equations, while the evolution of $\psi_{t}$ is simply driven by $\left(\chi_{t}, \phi_{t}\right)$ without any retroaction.

In the uncoupled case $\kappa=0$, we simply have $\dot{\phi}=1$ while $\chi$ and $\psi$ are constant. Taking into account the initial condition $U_{0}=\mathbb{1}$, we recover the fact that

$$
U_{t}=\left(\begin{array}{cc}
\mathrm{e}^{-\mathrm{i} t / 2} & 0 \\
0 & \mathrm{e}^{\mathrm{i} t / 2}
\end{array}\right)
$$

\subsection{Invariant measure}

We first prove existence and uniqueness of an invariant measure for the process $U_{t}$, under a rather general assumption on the noise $Z_{t}$.

Assumption 2.2. $\left\{Z_{t}\right\}_{t \geqslant 0}$ is a stationary, ergodic, real or complex-valued Markov process on the (canonical) probability space $\left(\Omega, \mathcal{F},\left\{\mathcal{F}_{t}\right\}_{t \geqslant 0}, \mathbb{P}\right)$ of the Brownian motion $\left\{W_{t}\right\}_{t \geqslant 0}$, defined by the Itô SDE (2.4), admitting the unique invariant probability measure $\nu$.

This assumption is satisfied if the drift term $f$ in the SDE (2.4) is sufficiently confining. For instance, in the real case, it is sufficient to assume that $-Z f(Z)$ grows at least quadratically as $|Z| \rightarrow \infty$, and that $g(Z)$ satisfies some ellipticity conditions.

Recall that the Haar measure of a compact Lie group $G$ is the unique probability measure $\mu$ on $G$ such that $\mu(g B)=\mu(B)$ for all Borel sets $B \subset G$. A computation of the

\footnotetext{
${ }^{2}$ Hence $(\phi, \psi)$ belong to a twisted two-torus (because $\left.u(\chi, \phi+2 \pi, \psi)=u(\chi, \phi, \psi+\pi)\right)$, but this will be of no concern to us.
} 
Jacobian of the transformation (2.13) shows that in our system of coordinates, the Haar measure is given by

$$
\mu(\mathrm{d} g)=\frac{1}{4 \pi^{2}} \sin (2 \chi) \mathrm{d} \chi \mathrm{d} \phi \mathrm{d} \psi .
$$

Theorem 2.3. Assume Assumption 2.2 holds. Then for any $\kappa \neq 0$ the product measure $\nu \otimes \mu$ is the unique invariant probability measure of the process $\left(Z_{t}, U_{t}\right)$.

The proof is given in Section 3. The fact that $\nu \otimes \mu$ is invariant is checked by a straightforward computation, while its uniqueness is proved using a control argument and the Stroock-Varadhan theorem.

Remark 2.4. Introducing a variable $\rho=\sin ^{2}(\chi)$ allows to rewrite the system in such a way that the invariant density is uniform. We will use this fact later on in the proof.

Theorem 2.3 leads to the following observations in terms of the physics of the model. Let $| \pm\rangle$ denote the canonical basis vectors of $\mathcal{H}$, i.e., the "spin up" and "spin down" states. Assume for instance that the system is prepared in the $|-\rangle$ state at time 0. Measuring the spin at time $t$, we will get $-1 / 2$ with probability

$$
\left|\left\langle-\left|U_{t}\right|-\right\rangle\right|^{2}=\cos ^{2} \chi_{t}=1-\rho_{t}
$$

and $+1 / 2$ with probability

$$
\left|\left\langle+\left|U_{t}\right|-\right\rangle\right|^{2}=\sin ^{2} \chi_{t}=\rho_{t}
$$

We should beware not to mix two different notions of probability. The expression (2.17) represents the probability of a quantum measurement yielding a certain value, for a given realisation $\omega \in \Omega$ of the noise. This is independent of the probability distribution on path space of the stochastic process $Z_{t}$. If the process $U_{t}$ were in the stationary state $\mu$, the expected probability of measuring a spin $+1 / 2$ would be given by

$$
\mathbb{E}^{\mu}\left[\sin ^{2} \chi\right]=\int_{0}^{2 \pi} \int_{0}^{2 \pi} \int_{0}^{\pi / 2} \sin ^{2} \chi \frac{1}{4 \pi^{2}} \sin (2 \chi) \mathrm{d} \chi \mathrm{d} \phi \mathrm{d} \psi=\frac{1}{2} .
$$

Also, we would have for any $b \in[0,1]$

$$
\mathbb{P}^{\mu}\left\{\left|\left\langle+\left|U_{t}\right|-\right\rangle\right|^{2} \leqslant b\right\}=\int_{0}^{2 \pi} \int_{0}^{2 \pi} \int_{\left\{\chi: \sin ^{2} \chi \leqslant b\right\}} \frac{1}{4 \pi^{2}} \sin (2 \chi) \mathrm{d} \chi \mathrm{d} \phi \mathrm{d} \psi=b .
$$

However, since by definition $U_{0}=\mathbb{1}$, the system cannot be in the stationary state $\mu$ at any given finite time $t$. At best, it can approach $\mu$ exponentially fast as time increases to ensure such a behaviour, we have to show the existence of a spectral gap. We shall do this under a more restrictive assumption on the noise term.

\subsection{Convergence to the invariant measure}

Assumption 2.5. The process $\left\{Z_{t}\right\}_{t \geqslant 0}$ is the real-valued stationary Ornstein-Uhlenbeck process defined by the SDE (2.5). In other words,

$$
Z_{t}=Z_{0} \mathrm{e}^{-\gamma t}+\sigma \int_{0}^{t} \mathrm{e}^{-\gamma(t-s)} \mathrm{d} W_{s}
$$

where $Z_{0}$ is a centred, Gaussian random variable, of variance $\sigma^{2} / 2 \gamma$, which is independent of the Brownian motion $\left\{W_{t}\right\}_{t \geqslant 0}$. 
Then the invariant measure $\nu$ is also Gaussian, centred, of variance $\sigma^{2} / 2 \gamma$. The parameter $\sigma^{2}$ can be interpreted as the temperature of the heat bath creating the noise, while $\gamma$ represents the rate of decay of correlations in the bath.

The assumption that $Z_{t}$ be real is not essential, but it simplifies the notations. In effect, we have to study the SDE

$$
\begin{aligned}
\mathrm{d} Z_{t} & =-\gamma Z_{t} \mathrm{~d} t+\sigma \mathrm{d} W_{t}, \\
\mathrm{~d} \chi_{t} & =\kappa Z_{t} \sin \phi_{t} \mathrm{~d} t, \\
\mathrm{~d} \phi_{t} & =\left(1+\frac{2 \kappa Z_{t} \cos \phi_{t}}{\tan 2 \chi_{t}}\right) \mathrm{d} t, \\
\mathrm{~d} \psi_{t} & =-\frac{\kappa Z_{t} \cos \phi_{t}}{\sin 2 \chi_{t}} \mathrm{~d} t,
\end{aligned}
$$

with initial condition $\left(\chi_{0}, \phi_{0}\right)=(0,0)$. The last equation, for $\mathrm{d} \psi_{t}$, is not really important for the dynamics, and has no measurable effect on physics either, $\psi_{t}$ having only an influence on the phase of matrix elements of $U_{t}$.

With the process $X_{t}=\left(Z_{t}, \chi_{t}, \phi_{t}, \psi_{t}\right)$ we associate in the usual way the Markov semigroup $T_{t}: \varphi(\cdot) \mapsto \mathbb{E}\left[\varphi\left(X_{t}\right) \mid X_{0}=\cdot\right]$. Its infinitesimal generator is the differential operator

$$
L=L_{Z}+\kappa Z \sin \phi \frac{\partial}{\partial \chi}+\left(1+\frac{2 \kappa Z \cos \phi}{\tan 2 \chi}\right) \frac{\partial}{\partial \phi}-\frac{\kappa Z \cos \phi}{\sin 2 \chi} \frac{\partial}{\partial \psi},
$$

where

$$
L_{Z}=-\gamma Z \frac{\partial}{\partial Z}+\frac{\sigma^{2}}{2} \frac{\partial^{2}}{\partial Z^{2}}
$$

is the generator of the Ornstein-Uhlenbeck process. The invariant measure $\nu \otimes \mu$ is an eigenfunction of the adjoint $L^{*}$ of $L$, with eigenvalue 0 . It is known that $L_{Z}$ has discrete spectrum, with real, nonpositive eigenvalues $-n \gamma, n \in \mathbb{N}_{0}$ (the eigenfunctions are Hermite polynomials). Since the other variables $\chi$ and $\phi$ live in a compact set, $L$ also has discrete spectrum. Moreover, Theorem 2.3 shows that 0 is a simple eigenvalue of $L$.

Theorem 2.6. Assume that Assumption 2.5 holds. Then for sufficiently small $\kappa \sigma$, there exists a constant $c$, independent of $\gamma, \kappa$ and $\sigma$, such that all eigenvalues of $L$ except 0 have a real part bounded above by 3

$$
-\frac{c}{T_{\gamma, \kappa \sigma}} \quad \text { where } T_{\gamma, \kappa \sigma}=\frac{1+\gamma^{2}}{(\kappa \sigma)^{2}} \vee \frac{1}{\gamma} .
$$

The proof is given in Section 4. It relies on standard second-order perturbation theory.

Theorem 2.6 shows that the distribution of the actual process $U_{t}$, starting with initial condition $\mathbb{1}$, converges exponentially fast to $\mu$, with rate $c / T_{\gamma, \kappa \sigma}$. Thus the expected (quantum) probability to measure a value $+1 / 2$ for the spin, when it starts in the "down" state satisfies

$$
\mathbb{E}^{\delta_{1}}\left[\sin ^{2} \chi_{t}\right]=\frac{1}{2}\left[1-\mathcal{O}\left(\mathrm{e}^{-c t / T_{\gamma, \kappa \sigma}}\right)\right] .
$$

Hence for times $t \gg T_{\gamma, \kappa \sigma}$, one can expect that a measurement of the spin will yield $-1 / 2$ or $+1 / 2$ with probability $1 / 2$ - provided the result is averaged over many realisations of the noise.

Regarding the dependence of the result on the correlation decay rate $\gamma$, we can distinguish two asymptotic regimes:

\footnotetext{
${ }^{3}$ For two real numbers $a, b, a \vee b$ denotes the maximum of $a$ and $b$ and $a \wedge b$ denotes the minimum of $a$ and $b$.
} 


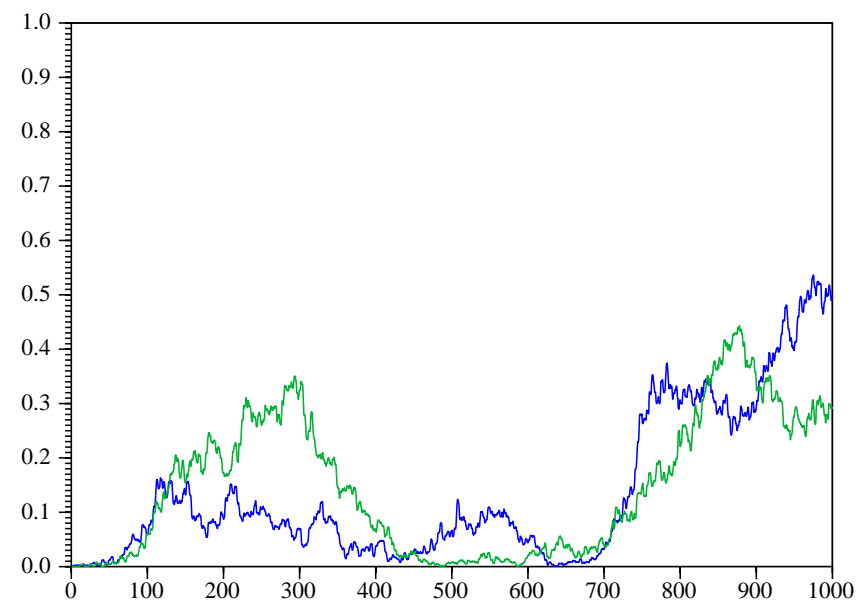

FIgURE 1. The probability $\left|\left\langle-\left|U_{t}\right|+\right\rangle\right|^{2}=\sin ^{2}\left(\chi_{t}\right)$ of measuring a transition from spin $-1 / 2$ to $+1 / 2$ as a function of time $t$, for two different realisations of the noise. Parameter values are $\gamma=1, \kappa=1$ and $\sigma=0.03$, so that the relaxation time $T_{\gamma, \kappa \sigma}$ to the invariant measure is of order 2000 .

- For $\gamma \ll 1$, the correlations in the Ornstein-Uhlenbeck process decay very slowly, in fact $Z_{t}$ resembles a Brownian motion. As a result, the relaxation time $T_{\gamma, \kappa \sigma}$ becomes very large.

- For $\gamma \gg \kappa \sigma$, the correlations in the Ornstein-Uhlenbeck process decay quickly, so that $Z_{t}$ resembles white noise. Then the relaxation time $T_{\gamma, \kappa \sigma}$ also becomes large.

Relaxation to equilibrium is fastest when $\gamma \simeq(\kappa \sigma)^{2}$. Then $T_{\gamma, \kappa \sigma}$ has order $1 /(\kappa \sigma)^{2}$.

\subsection{Diffusion approximation for the transition probability}

The probability of measuring a "spin up" at time $t$, if the system is prepared in the "spin down" state at time 0 , for a given realisation $\omega$ of the noise, is given by

$$
\rho_{t}(\omega)=\left|\left\langle-\left|U_{t}(\omega)\right|+\right\rangle\right|^{2}=\sin ^{2}\left(\chi_{t}(\omega)\right) .
$$

While the spectral gap result Theorem 2.6 yields a control on the average of $\rho_{t}$ over many realisations $\omega$ of the noise, it does not describe its time-evolution very precisely. In this section we provide a more precise description of the dynamics by giving pathwise estimates on $\rho_{t}(\omega)$, in the form of first-passage times. This is done by obtaining an approximately closed effective equation for $\rho_{t}$, which is the only physically measurable quantity in the system. The methods used are partly adapted from those presented in [BG06].

Figure 1 shows two sample paths $t \mapsto \rho_{t}(\omega)$, obtained for two different realisations $W_{t}(\omega)$ of the Brownian motion driving $Z_{t}$. For the parameter values $\kappa=\gamma=1$ and $\sigma=0.03$ used in Figure 1, this probability remains larger than $1 / 2$ for all times up to 1000. Hence the system is still far from its invariant measure.

Figure 2 shows two sample paths $t \mapsto \rho_{t}(\omega)$ in a case with larger noise intensity $\sigma=0.1$. Now the sample paths have enough time to explore all of phase space, indicating that the system has reached equilibrium. Note however that for any given realisation of the noise, a spin measurement made after a sufficiently long time may still yield $-1 / 2$ with any probability. Only by making repeated measurements in the course of time would one obtain an average probability close to $1 / 2$ - assuming that the measurements do not affect the state of the system, which of course they do. 


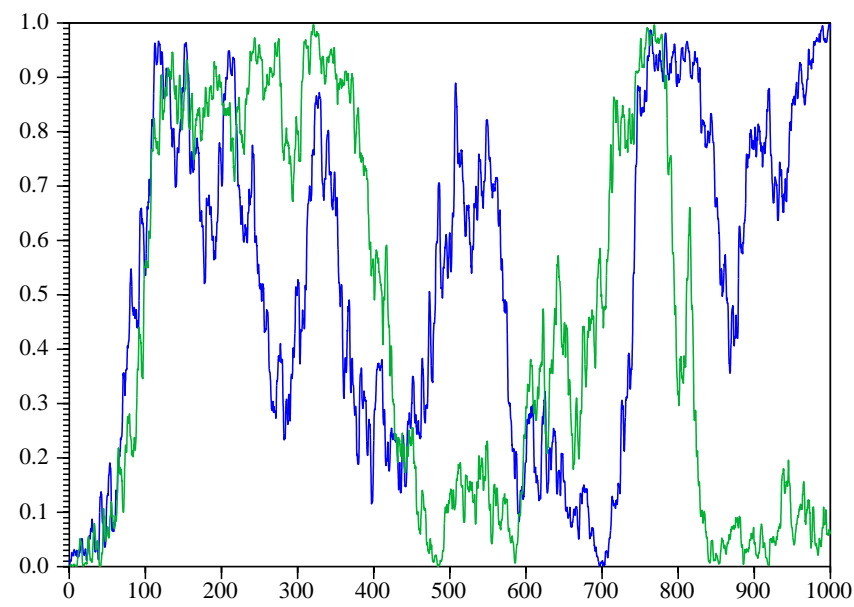

FIgURE 2. The probability $\left|\left\langle-\left|U_{t}\right|+\right\rangle\right|^{2}=\sin ^{2}\left(\chi_{t}\right)$ of measuring a transition from spin $-1 / 2$ to $+1 / 2$ as a function of time $t$, for two different realisations of the noise. Parameter values are $\gamma=1, \kappa=1$ and $\sigma=0.1$, so that the relaxation time $T_{\gamma, \kappa \sigma}$ to the invariant measure is of order 200 .

Let us now describe the derivation of the effective equation for $\rho_{t}$. Recall that the uniform measure is invariant when the system is written in the variables $(\rho, \phi, \psi)$. In order to exploit symmetries of the problem, it is convenient to use the variable $y=2 \rho-1=$ $-\cos 2 \chi \in[-1,1]$ instead of $\rho$. We are led to consider the SDE on $\mathbb{R} \times[-1,1] \times \mathbb{S}^{1}$

$$
\begin{aligned}
\mathrm{d} Z_{t} & =-\gamma Z_{t} \mathrm{~d} t+\sigma \mathrm{d} W_{t}, \\
\mathrm{~d} y_{t} & =2 \kappa Z_{t} \sqrt{1-y_{t}^{2}} \sin \phi_{t} \mathrm{~d} t, \\
\mathrm{~d} \phi_{t} & =\left[1-2 \kappa Z_{t} \frac{y_{t}}{\sqrt{1-y_{t}^{2}}} \cos \phi_{t}\right] \mathrm{d} t .
\end{aligned}
$$

Observe that if $\kappa$ (or $\sigma$ ) is small, then this system displays several distinct timescales. While $\phi_{t}$ will be close to $t, y_{t}$ can at best grow like $\kappa t$. In fact, if $Z$ were constant, one can see that the system (2.28) restricted to $(y, \phi)$ is conservative, that is, there exists a constant of motion $K(y, \phi)=y+\mathcal{O}(\kappa)$. The fact that $Z_{t}$ is time-dependent actually destroys this invariance, but in a very soft way.

The usual way to obtain an effective diffusion equation for $y$ (or for the constant of motion $K(y, \phi))$ is to average the right-hand side of (2.28) over the fast variable $\phi$ [FW04]. However, in the present case this average is zero, so that one has to go beyond the usual averaging procedure.

It turns out that one can construct a new variable $\bar{y}=y+\kappa w(Z, y, \phi)$ such that

$$
\mathrm{d} \bar{y}_{t} \simeq-\frac{4 \sigma^{2} \gamma}{1+\gamma^{2}} Z_{t}^{2} \bar{y}_{t} \mathrm{~d} t+\frac{2 \kappa \sigma}{\sqrt{1+\gamma^{2}}} \sqrt{1-\bar{y}_{t}^{2}} \cos \left(\phi_{t}+\theta\right) \mathrm{d} W_{t},
$$

where $\theta$ is a constant phase shift (see Proposition 5.1 for a precise formulation). The variance of the noise term grows like $t$ times the square of the coefficient of $\mathrm{d} W_{t}$. Since $\phi_{t}$ rotates rapidly, we expect that $\cos ^{2}\left(\phi_{t}+\theta\right)$ can be approximated by $1 / 2$. In addition, $Z_{t}^{2}$ is rapidly fluctuating with average $\sigma^{2} / 2 \gamma$. We thus expect that

$$
\mathrm{d} \bar{y}_{t} \simeq-\frac{2(\kappa \sigma)^{2}}{1+\gamma^{2}} \bar{y}_{t} \mathrm{~d} t+\sqrt{\frac{2(\kappa \sigma)^{2}}{1+\gamma^{2}}} \sqrt{1-\bar{y}_{t}^{2}} \mathrm{~d} W_{t},
$$


which, after rescaling time by a factor $T_{\gamma, \kappa \sigma}$ reduces to

$$
\mathrm{d} \bar{y}_{t} \simeq-\bar{y}_{t} \mathrm{~d} t+\sqrt{1-\bar{y}_{t}^{2}} \mathrm{~d} W_{t} .
$$

The sample paths of this system explore phase space in a rescaled time of order 1.

The approximation (2.30) is of course not rigorous. But based on the above intuition, one can prove the following result on the time needed for $y_{t}$ to explore phase space.

Theorem 2.7. Assume that Assumption 2.5 holds. Let

$$
\tau(y)=\inf \left\{t>0: y_{t}>y\right\}
$$

be the random first-passage time of $y_{t}$ at the value $y$. Then there is a function $e(y)$, independent of $\gamma, \sigma$ and $\kappa$ and bounded for $y<1$, such that

$$
\mathbb{E}[\tau(y)] \leqslant e(y) T_{\gamma, \kappa \sigma}=e(y)\left[\frac{1+\gamma^{2}}{(\kappa \sigma)^{2}} \vee \frac{1}{\gamma}\right] .
$$

Furthermore, $\mathbb{P}\{\tau(y)>t\}$ decays exponentially with rate of order $1 / e(y) T_{\gamma, \kappa \sigma}$.

The proof is given in Section 5. It uses comparison inequalities for the stochastic differential equation satisfied by $\bar{y}_{t}$. The main difficulty is that one obtains different approximations in different regions of phase space, which have then to be patched together. This is done with the help of renewal equations, solved by Laplace transforms.

The time $T_{\gamma, \kappa \sigma}$ plays the rôle of a typical exploration time. It has value 2000 in Figure 1 and 200 in Figure 2, The function $e(y)$ may diverge as $y \nearrow 1$. This is due to the fact that $y=1$ (i.e., $\chi=\pi / 2$ ) corresponds to a single curve in $\mathrm{SU}(2)$, depending only on $\phi / 2-\psi$, a too small set to hit.

\section{Remark 2.8.}

1. Theorem 2.7 does not follow from Theorem 2.6. For instance, the Ornstein-Uhlenbeck process $Z_{t}$ has a spectral gap $\gamma$, but the process takes exponentially long times of order $\mathrm{e}^{\gamma h / \sigma^{2}}$ to reach values of order $h$.

2. Conversely, Theorem 2.6 partially follows from Theorem 2.7, namely for real eigenvalues. Indeed, given a subset $\mathcal{D}$ of phase space, consider the following boundary value problem:

$$
\begin{aligned}
-L \varphi & =\lambda \varphi & & \text { for } x \in \mathcal{D}, \\
\varphi & =g & & \text { for } x \in \partial \mathcal{D} .
\end{aligned}
$$

For Dirichlet boundary conditions $g=0$, it is known that the eigenvalue $\bar{\lambda}(\mathcal{D})$ of smallest real part of this problem is real and simple [Jen12, PW66]. A classical result due to Donsker and Varadhan [DV76] states that

$$
\bar{\lambda}(\mathcal{D}) \geqslant \frac{1}{\sup _{x \in D} \mathbb{E}^{x}\left[\tau_{\mathcal{D}}\right]},
$$

where $\tau_{\mathcal{D}}=\inf \left\{t>0: x_{t} \notin \mathcal{D}\right\}$ is the first-exit time from $\mathcal{D}$. Now if $\lambda<\bar{\lambda}(\mathcal{D})$, the boundary value problem (2.34) has a unique solution for every boundary condition $g$, given by

$$
\varphi(x)=\mathbb{E}^{x}\left[\mathrm{e}^{\lambda \tau_{\mathcal{D}}} g\left(x_{\tau_{\mathcal{D}}}\right)\right] .
$$

Any eigenfunction of $L$ corresponding to a nonzero real eigenvalue must change sign. Thus taking $\mathcal{D}=\{x: \varphi(x)>0\}$ would yield $g=0$, and thus also $\varphi(x)=0 \forall x \in \mathcal{D}$, a contradiction. Hence $L$ cannot have any real eigenvalue smaller than $\bar{\lambda}(\mathcal{D})$, except 0 . 


\section{Existence and uniqueness of the invariant measure}

\subsection{Invariance of the Haar measure}

With the semigroup $T_{t}$ of the Markov process, we associate the dual semigroup $S_{t}$, acting on $\sigma$-finite measures $\mu$ according to

$$
\left(S_{t} \mu\right)(B)=\int \mathbb{P}\left\{X_{t} \in B \mid X_{0}=x\right\} \mu(\mathrm{d} x)=: \mathbb{P}^{\mu}\left\{X_{t} \in B\right\} .
$$

Its infinitesimal generator is the adjoint $L^{*}$ of the generator $L$. If $\mu$ has density $\rho$ with respect to the Lebesgue measure, then

$$
L^{*} \rho=L_{Z}^{*} \rho-\kappa Z \sin \phi \frac{\partial \rho}{\partial \chi}-\frac{\partial}{\partial \phi}\left[1+\left(\frac{2 \kappa Z \cos \phi}{\tan 2 \chi}\right) \rho\right]+\frac{\kappa Z \cos \phi}{\sin 2 \chi} \frac{\partial \rho}{\partial \psi} .
$$

Now in our case, the measure which is claimed to be invariant is the product measure $\nu \otimes \mu$, where $\mu$ has density $\sin (2 \chi) / 4 \pi^{2}$. Since $\nu$ is invariant for the process $Z_{t}, L_{Z}^{*} \nu=0$. It is then immediate to check that $L^{*} \rho=0$.

\subsection{Proof of Theorem 2.3}

We would like to show that $\nu \otimes \mu$ is the unique invariant measure of the process $\left(Z_{t}, U_{t}\right)$. Since the generator $L$ of the process is very far from uniformly elliptic, we cannot use standard ellipticity (or even hypo-ellipticity) arguments. To circumvent this difficulty, we shall combine an argument of control theory with the Stroock-Varadhan support theorem.

We start by writing the joint system (2.4),(2.14) as

$$
\begin{aligned}
\mathrm{d} Z_{t} & =f\left(Z_{t}\right) \mathrm{d} t+g\left(Z_{t}\right) \mathrm{d} W_{t}, \\
\mathrm{~d} x_{t} & =\left[b_{0}\left(x_{t}\right)+\kappa X_{t} b_{1}\left(x_{t}\right)+\kappa Y_{t} b_{2}\left(x_{t}\right)\right] \mathrm{d} t,
\end{aligned}
$$

where $Z_{t}=X_{t}+\mathrm{i} Y_{t}, x_{t}=\left(\chi_{t}, \phi_{t}, \psi_{t}\right)$ and

$$
\begin{aligned}
& b_{0}(x)=(0,1,0), \\
& b_{1}(x)=\left(\sin \phi, \frac{2 \cos \phi}{\tan 2 \chi},-\frac{\cos \phi}{\sin 2 \chi}\right), \\
& b_{2}(x)=\left(\cos \phi,-\frac{2 \sin \phi}{\tan 2 \chi}, \frac{\sin \phi}{\sin 2 \chi}\right) .
\end{aligned}
$$

We denote by $P_{t}\left(\left(Z_{0}, x_{0}\right), B\right)=\mathbb{P}^{\left(Z_{0}, x_{0}\right)}\left\{\left(Z_{t}, x_{t}\right) \in B\right\}$ the transition probabilities of the Markov process with initial condition $\left(Z_{0}, x_{0}\right)$. With (3.3) we associate the control system

$$
\begin{aligned}
\dot{Z} & =f(Z)+g(Z) u(t), \\
\dot{x} & =b_{0}(x)+\kappa X b_{1}(x)+\kappa Y b_{2}(x),
\end{aligned}
$$

where $u: \mathbb{R}_{+} \rightarrow \mathbb{R}$ is a piecewise constant function. The accessible set from an initial condition $\left(Z_{0}, x_{0}\right)$ is the set

$$
A_{t}\left(Z_{0}, x_{0}\right)=\left\{(Z, x): \exists u:[0, t] \rightarrow \mathbb{R},(Z(0), x(0))=\left(Z_{0}, x_{0}\right),(Z(t), x(t))=(Z, x)\right\} .
$$

The Stroock-Varadhan support theorem [SV72a, SV72b] states that

$$
\operatorname{supp} P_{t}\left(\left(Z_{0}, x_{0}\right), \cdot\right)=\overline{A_{t}\left(Z_{0}, x_{0}\right)},
$$

that is, that $P_{t}\left(\left(Z_{0}, x_{0}\right), B\right)>0$ for any open set $B \subset A_{t}\left(Z_{0}, x_{0}\right)$. 
Proposition 3.1. For any $\kappa \neq 0$, any initial condition $\left(Z_{0}, x_{0}\right)$ and any $t>0$, the closure of the accessible set $A_{t}\left(Z_{0}, x_{0}\right)$ is the whole phase space $\mathbb{C} \times[0, \pi / 2] \times \mathbb{T}^{2}$.

Proof: We first observe that by Assumption 2.2, the process $Z_{t}$ is controllable, that is, for any $t>0$ and any trajectory $\left\{Z_{s}\right\}_{0 \leqslant s \leqslant t}$, one can find a piecewise constant control $u$ such that $f\left(Z_{t}\right)+g\left(Z_{t}\right) u(t)$ approximates $\dot{Z}(t)$ arbitrarily closely. As a consequence, we can slightly simplify the problem by considering the three-dimensional control problem

$$
\dot{x}=b_{0}(x)+\kappa b_{1}(x) u_{1}(t)+\kappa b_{2}(x) u_{2}(t) .
$$

It is in fact sufficient to discuss the particular case $Z_{t} \in \mathbb{R}$, that is, $u_{2}=0$, the other cases being treated similarly.

Let $\Gamma=[0, \pi / 2] \times \mathbb{T}^{2}$. The accessibility algebra $\mathcal{A}(x)$ of a point $x \in \Gamma$ is the smallest sub-algebra of the Lie algebra of vector fields over $\Gamma$ containing $b_{0}$ and $b_{1}$. It is generated by all iterated Lie brackets of $b_{0}$ and $b_{1}$. We identify each vector field $b_{i}$ with an operator $A_{i}=\sum_{j} b_{i}^{j} \partial_{j}$. Then Lie brackets of $b_{i}$ are identified with commutators of $A_{i}$. Now straightforward computations show that

$$
\begin{aligned}
& {\left[A_{0}, A_{1}\right]=A_{2},} \\
& {\left[A_{0}, A_{2}\right]=-A_{1},} \\
& {\left[A_{1}, A_{2}\right]=4 A_{0} .}
\end{aligned}
$$

As a consequence, the accessibility algebra $\mathcal{A}(x)$ is generated by the vector fields $b_{0}, b_{1}$ and $b_{2}$. Since

$$
\operatorname{det}\left\{b_{0}, b_{1}, b_{2}\right\}=-\frac{1}{\sin 2 \chi},
$$

we conclude that $\operatorname{span}[\mathcal{A}(x)]$ has dimension 3 for any $x$, except possibly for $\chi \in\{0, \pi / 2\}$.

In order to treat the case $\chi=\pi / 2$, we use the representation (2.11) of the system. $\chi=\pi / 2$ corresponds to $x_{3}=x_{4}=0$. Near a point $\left(x_{1}^{\star}, x_{2}^{\star}, 0,0\right)$, the Lie group $\mathrm{SU}(2)$ can be parametrised by $\left(y_{2}, y_{3}, y_{4}\right)=\left(x_{2}-x_{2}^{\star}, x_{3}, x_{4}\right)$, the remaining variable $x_{1}$ being expressed in terms of the other variables with the help of Relation (2.10). Writing the vector field in variables $\left(y_{2}, y_{3}, y_{4}\right)$ and computing Lie brackets, we obtain again that the accessibility algebra is generated by three vector fields and has dimension 3 . The case $\chi=0$ is treated similarly.

Whenever $\operatorname{span}[\mathcal{A}(x)]$ has full dimension, a standard result from control theory (see [NvdS90, Chapter 3]) states that $A_{t}(x)$ contains an open, non-empty neighbourhood of $x$. Since this is true for all $x$, we have proved that $\overline{\mathcal{A}(x)}=\Gamma$ for all $x \in \Gamma$.

Proof of Theorem 2.3. Let $\mathcal{M}$ denote the convex set of all invariant measures of the process. With every invariant measure $\mu \in \mathcal{M}$ we can associate a stationary Markov process $\mathbb{P}^{\mu}$. It is known that a measure $\mu \in \mathcal{M}$ is extremal if and only if the associated dynamical system is ergodic. Thus if $\mathcal{M}$ contains two measures $\mu_{1} \neq \mu_{2}$, the measure $\mu=\frac{1}{2}\left(\mu_{1}+\mu_{2}\right)$ is invariant but not ergodic.

The previous proposition implies, with Stroock-Varadhan's support theorem, that $P_{t}\left(\left(Z_{0}, x_{0}\right), B\right)>0$ for any open $B \subset \mathbb{C} \times[0, \pi / 2] \times \mathbb{T}^{2}$. If $\mu$ were not ergodic, we could find an open set $B$, with $0<\mu(B)<1$, such that $P_{t}\left(\left(Z_{0}, x_{0}\right), B\right)=1$ for $\mu$-almost all $\left(Z_{0}, x_{0}\right) \in B$ and $P_{t}\left(\left(Z_{0}, x_{0}\right), B^{c}\right)=0$ for $\mu$-almost all $\left(Z_{0}, x_{0}\right) \in B^{c}$, a contradiction. Hence $\mu$ must be ergodic, and thus $\mathcal{M}$ contains only one measure. 


\section{Convergence to the invariant measure}

Let us first remark that since the Ornstein-Uhlenbeck process $Z_{t}$ is proportional to $\sigma$, and enters in the dynamics only through the coupling constant $\kappa$, all results will only depend on the product $\kappa \sigma$. We may thus choose one of the parameters at our convenience, keeping the product fixed. In this section, we consider that in fact $\sigma=1$, but keep writing $\sigma$ for more clarity.

\subsection{Proof of Theorem 2.6}

In order to exploit the fact that the invariant measure is uniform when taking variables $\left(y=2 \sin ^{2} \chi-1, \phi, \psi\right)$, we consider, instead of the infinitesimal generator (2.23) of our diffusion process, the equivalent operator

$$
L=L_{Z}+\kappa Z \sqrt{1-y^{2}} \sin \phi \frac{\partial}{\partial y}+\left[1-\frac{2 \kappa Z y}{\sqrt{1-y^{2}}} \cos \phi\right] \frac{\partial}{\partial \phi}-\frac{\kappa Z \cos \phi}{\sqrt{1-y^{2}}} \frac{\partial}{\partial \psi} .
$$

We can write this generator as $L=L_{0}+\mathrm{i} \kappa L_{1}$, where $L_{0}=L_{Z}+\partial / \partial \phi$. The operator $L_{Z}$ is self-adjoint in $L^{2}(\mathbb{R}, \nu(\mathrm{d} Z))$, and has simple eigenvalues $-n \gamma, n \in \mathbb{N}_{0}$. Its eigenfunctions are (properly normalised) Hermite polynomials, which we denote $h_{n}(Z)$. In particular, we have

$$
h_{0}(Z)=1, \quad h_{1}(Z)=\frac{\sqrt{2 \gamma}}{\sigma} Z .
$$

An integration by parts shows that

$$
L_{1}:=-\mathrm{i} Z \sqrt{1-y^{2}} \sin \phi \frac{\partial}{\partial y}+\frac{2 \mathrm{i} Z y}{\sqrt{1-y^{2}}} \cos \phi \frac{\partial}{\partial \phi}+\frac{\mathrm{i} Z \cos \phi}{\sqrt{1-y^{2}}} \frac{\partial}{\partial \psi}
$$

is also self-adjoint in $L^{2}\left([-1,1] \times \mathbb{T}^{2}, \mathrm{~d} y \mathrm{~d} \phi \mathrm{d} \psi\right)$. We may choose an orthonormal basis of $L^{2}\left(\mathbb{R} \times[-1,1] \times \mathbb{T}^{2},(\nu \otimes \mu)(\mathrm{d} x)\right)$ given by

$$
|n, p, k, r\rangle=h_{n}(Z) f_{p}(y) \mathrm{e}^{\mathrm{i} k \phi} \mathrm{e}^{\mathrm{i} r \psi},
$$

where $\left\{f_{p}(y)\right\}_{p \in \mathbb{Z}}$ is an arbitrary orthonormal basis of $L^{2}([-1,1], \mathrm{d} y)$ (a Fourier basis will do). Since

$$
L_{0}|n, p, k, r\rangle=\lambda_{n, k}^{0}|n, p, k, r\rangle, \quad \lambda_{n, k}^{0}=-n \gamma+\mathrm{i} k,
$$

we see that $L_{0}$ has infinitely many eigenvalues on the imaginary axis, which are infinitely degenerate. We can nevertheless apply standard time-independent perturbation theory to second order in $\kappa$ (see for instance [LL58]). Expanding eigenfunctions in the basis (4.4), then expanding everything in $\kappa$ and projecting on the basis functions shows that the perturbed eigenfunctions have the form

$$
|n, p, k, r\rangle+\mathrm{i} \kappa \sum_{m, q, l, s} \frac{\left\langle m, q, l, s\left|L_{1}\right| n, p, k, r\right\rangle}{\lambda_{n, k}^{0}-\lambda_{m, l}^{0}}|m, q, l, s\rangle+\mathcal{O}\left(\kappa^{2}\right),
$$

where the sum runs over all $(m, q, l, s) \neq(n, p, k, r)$ such that $\left\langle m, q, l, s\left|L_{1}\right| n, p, k, r\right\rangle \neq 0$. The perturbed eigenvalues have the form

$$
\begin{aligned}
\lambda_{n, p, k, r}= & \lambda_{n, k}^{0}+\mathrm{i} \kappa\left\langle n, p, k, r\left|L_{1}\right| n, p, k, r\right\rangle \\
& -\kappa^{2} \sum_{m, q, l, s} \frac{\left|\left\langle m, q, l, s\left|L_{1}\right| n, p, k, r\right\rangle\right|^{2}}{\lambda_{n, k}^{0}-\lambda_{m, l}^{0}}+\mathcal{O}\left(\kappa^{3}\right) .
\end{aligned}
$$


Note the minus sign due to the fact that the perturbation term i $L_{1}$ is anti-hermitian. We now have to compute matrix elements of $L_{1}$ in the chosen basis. We are however only interested in matrix elements involving $n=0$, which give perturbations of eigenvalues on the imaginary axis. Using (4.2), we get

$$
-\mathrm{i} Z \sqrt{1-y^{2}} \sin \phi \frac{\partial}{\partial y}|0, p, k, r\rangle=\frac{\sigma}{\sqrt{2 \gamma}} h_{1}(Z) \sqrt{1-y^{2}} f_{p}^{\prime}(y) \frac{1}{2}\left[\mathrm{e}^{\mathrm{i}(k+1) \phi}-\mathrm{e}^{\mathrm{i}(k-1) \phi}\right] \mathrm{e}^{\mathrm{i} r \psi}
$$

showing that

$$
\left\langle m, q, l, s\left|-\mathrm{i} Z \sqrt{1-y^{2}} \sin \phi \frac{\partial}{\partial y}\right| 0, p, k, r\right\rangle=\frac{\sigma}{\sqrt{2 \gamma}} \delta_{m, 1} a_{q, p}\left[\delta_{l, k+1}-\delta_{l, k-1}\right] \delta_{s, r}
$$

where

$$
a_{q, p}=\int_{-1}^{1} \overline{f_{q}(y)} \sqrt{1-y^{2}} f_{p}^{\prime}(y) \mathrm{d} y .
$$

Proceeding in a similar way for the other terms of $L_{1}$, we get

$$
\left\langle m, q, l, s\left|L_{1}\right| 0, p, k, r\right\rangle=\frac{\sigma}{\sqrt{2 \gamma}} \delta_{m, 1}\left[\left(a_{q, p}+b_{q, p}\right) \delta_{l, k+1}+\left(-a_{q, p}+b_{q, p}\right) \delta_{l, k-1}\right] \delta_{s, r},
$$

where

$$
b_{q, p}(k, r)=\int_{-1}^{1} \overline{f_{q}(y)} \frac{k y+\frac{1}{2} r}{\sqrt{1-y^{2}}} f_{p}(y) \mathrm{d} y .
$$

The expression (4.11) of the matrix elements shows that the first-order correction to the eigenvalues vanishes. It also shows that the second-order correction term in (4.7) is welldefined, because the denominator never vanishes when the matrix element is nonzero. The expansion for the eigenvalue becomes

$$
\lambda_{0, p, k, r}=\mathrm{i} k-\frac{(\kappa \sigma)^{2}}{2 \gamma} \sum_{q}\left[\frac{\left|a_{q, p}+b_{q, p}(k, r)\right|^{2}}{\gamma+\mathrm{i}}+\frac{\left|a_{q, p}-b_{q, p}(k, r)\right|^{2}}{\gamma-\mathrm{i}}\right]+\mathcal{O}\left(\kappa^{3}\right) .
$$

In particular,

$$
\operatorname{Re} \lambda_{0, p, k, r}=-\frac{(\kappa \sigma)^{2}}{2\left(1+\gamma^{2}\right)} \sum_{q}\left[\left|a_{q, p}+b_{q, p}(k, r)\right|^{2}+\left|a_{q, p}-b_{q, p}(k, r)\right|^{2}\right]+\mathcal{O}\left(\kappa^{3}\right) .
$$

It remains to see that the sum over $q$ does not vanish. Being a sum of non-negative terms, it vanishes if and only if all its terms vanish, which happens if and only if $a_{q, p}=0$ and $b_{q, p}(k, r)=0$ for all $q$. Now for $(k, r) \neq(0,0), b_{q, p}(k, r)$ cannot vanish for all $q$, because the function $\left(k y+\frac{1}{2} r\right) f_{p}(y) / \sqrt{1-y^{2}}$ cannot be orthogonal to all basis functions. For $(k, r)=(0,0)$, we have $b_{q, p}(0,0)=0$. However in that case, $a_{q, p}=0$ for all $q$ if and only if $\sqrt{1-y^{2}} f_{p}^{\prime}(y)$ is identically zero, that is, only in the case $p=0$ where $f_{p}(y)$ is constant. But this corresponds to the zero eigenvalue of the invariant measure. 


\section{Diffusion approximation}

In this section, it will be more convenient to consider both $\kappa$ and $\sigma$ as small parameters.

\subsection{Averaging}

We consider the $\mathrm{SDE}$ on $\mathbb{R} \times[-1,1] \times \mathbb{S}^{1}$ given by

$$
\begin{aligned}
\mathrm{d} Z_{t} & =-\gamma Z_{t} \mathrm{~d} t+\sigma \mathrm{d} W_{t}, \\
\mathrm{~d} y_{t} & =2 \kappa Z_{t} \sqrt{1-y_{t}^{2}} \sin \phi_{t} \mathrm{~d} t, \\
\mathrm{~d} \phi_{t} & =\left[1-2 \kappa Z_{t} \frac{y_{t}}{\sqrt{1-y_{t}^{2}}} \cos \phi_{t}\right] \mathrm{d} t .
\end{aligned}
$$

For small $\kappa$, the variable $\phi_{t} \simeq t$ changes much faster than $y_{t}$, while $Z_{t}$ also fluctuates on timescales of order 1 . The philosophy of averaging tells us that the dynamics of $y_{t}$ should be close to the one obtained by averaging the right-hand side over all fast variables. However this average vanishes in the present case, so that we have to look into the averaging procedure in more detail.

Proposition 5.1. There exists a change of variable $y \mapsto \bar{y}$, with $\bar{y} \in[-1,1]$, such that

$$
\mathrm{d} \bar{y}_{t}=F\left(Z_{t}, \bar{y}_{t}, \phi_{t}\right) \mathrm{d} t+G\left(Z_{t}, \bar{y}_{t}, \phi_{t}\right) \mathrm{d} W_{t},
$$

where the drift term satisfies

$$
F(Z, \bar{y}, \phi)=-\frac{4 \kappa^{2} \gamma}{1+\gamma^{2}} Z^{2} \bar{y}+\mathcal{O}\left(\frac{\kappa^{3} \gamma Z^{3}}{\left(1+\gamma^{2}\right)^{2}}\right)
$$

and the diffusion term is of the form

$$
G(Z, \bar{y}, \phi)=2 \kappa \sigma \sqrt{\frac{1-\bar{y}^{2}}{1+\gamma^{2}}} \cos (\phi+\theta)+\mathcal{O}\left(\frac{\kappa^{2} \sigma Z}{1+\gamma^{2}}\right) .
$$

where $\theta=\arctan \gamma$.

Proof: Averaging amounts to looking for a $\phi$-periodic change of variables removing the dependence of $\mathrm{d} y_{t}$ on $\phi_{t}$. We thus set $\bar{y}=y+\kappa w(Z, y, \phi)$ and plug this into the equation, yielding

$$
\begin{aligned}
\mathrm{d} \bar{y}_{t}= & \kappa\left[Z_{t} \sqrt{1-y_{t}^{2}} \sin \phi_{t}-\gamma Z_{t} \frac{\partial w}{\partial Z}+\frac{\partial w}{\partial \phi}\right] \mathrm{d} t \\
& +2 \kappa^{2} Z_{t}\left[\sqrt{1-y_{t}^{2}} \sin \phi_{t} \frac{\partial w}{\partial y}-\frac{y_{t}}{\sqrt{1-y_{t}^{2}}} \cos \phi_{t} \frac{\partial w}{\partial \phi}\right] \mathrm{d} t+\kappa \sigma \frac{\partial w}{\partial Z} \mathrm{~d} W_{t}
\end{aligned}
$$

The choice

$$
w(Z, y, \phi)=\frac{2 Z}{1+\gamma^{2}} \sqrt{1-y^{2}}[\cos \phi+\gamma \sin \phi]
$$

allows to eliminate the first term in brackets, and yields

$$
\mathrm{d} \bar{y}_{t}=-\frac{4 \kappa^{2} \gamma}{1+\gamma^{2}} Z_{t}^{2} y_{t} \mathrm{~d} t+\frac{2 \kappa \sigma}{1+\gamma^{2}} \sqrt{1-y_{t}^{2}}\left[\cos \phi_{t}+\gamma \sin \phi_{t}\right] \mathrm{d} W_{t} .
$$

Finally, using the equality $\cos \phi+\gamma \sin \phi=\sqrt{1+\gamma^{2}} \cos (\phi+\theta)$ and replacing $y_{t}$ by $\bar{y}_{t}$ yields the above expressions for $\mathrm{d} \bar{y}_{t}$.

We see that the averaging transformation has created an effective drift term, fluctuating rapidly in time. 


\section{$5.2 \quad$ First-passage times}

We would like to estimate the expected first-passage time of $\bar{y}_{t}$ at any level $y<1$, and for the initial condition $\left(y_{0}, \phi_{0}\right)=(-1,0)$. We split this problem into several parts, estimating first the time needed to reach a level $-\delta_{1}<0$, then the level 0 , and finally all positive $y$. Also, in order to avoid difficulties due to $Z_{t}$ becoming very large, we will first work within the set $\left\{\omega:\left|Z_{t}\right| \leqslant 1 \forall t\right\}$, and deal later with the rare events that $\left|Z_{t}\right|$ becomes larger than 1. For this purpose, it is useful to introduce the notation

$$
\tau_{\mathcal{D}}=\inf \left\{t>0: x_{t} \notin \mathcal{D}\right\}
$$

for the first-exit time of $x_{t}=\left(Z_{t}, \bar{y}_{t}, \phi_{t}\right)$ from a set $\mathcal{D} \subset \mathbb{R} \times[-1,1] \times \mathbb{S}^{1}$. We denote by $\mathbb{P}^{x_{0}}$ the law of the process starting in some point $x_{0}=\left(Z_{0}, \bar{y}_{0}, \phi_{0}\right)$ at time 0 , and by $\mathbb{E}^{x_{0}}$ expectations with respect to this law. Note that we have to consider here deterministic initial conditions $Z_{0}$, and only at the end will we take expectations with respect to the initial distribution of $Z$. We also introduce the notation

$$
T^{\star}=\frac{1+\gamma^{2}}{(\kappa \sigma)^{2}} .
$$

The following lemma will allow us to reduce the problem to time intervals of fixed size $T$.

Lemma 5.2. Fix $T>0$ and let

$$
q(T)=\sup _{x_{0}^{\prime} \in \mathcal{D}} \mathbb{P}^{x_{0}^{\prime}}\left\{\tau_{\mathcal{D}}>T\right\}
$$

If $q(T)>0$, then

$$
\mathbb{P}^{x_{0}}\left\{\tau_{\mathcal{D}}>t\right\} \leqslant q(T)^{-1} \mathrm{e}^{-\log q(T)^{-1} t / T}
$$

holds for all $t>0$ and all $x_{0} \in \mathcal{D}$.

Proof: We set $t=n T+u$, with $n=\lfloor t / T\rfloor$. By the strong Markov property, we have

$$
\begin{aligned}
\mathbb{P}^{x_{0}}\left\{\tau_{\mathcal{D}}>t\right\} & \leqslant \mathbb{P}^{x_{0}}\left\{\tau_{\mathcal{D}}>n T\right\} \\
& \leqslant \mathbb{E}^{x_{0}}\left[1_{\left\{\tau_{\mathcal{D}}>(n-1) T\right\}} \mathbb{P}^{x_{(n-1) T}}\left\{\tau_{\mathcal{D}}>n T\right\}\right] \\
& \leqslant q(T) \mathbb{P}^{x_{0}}\left\{\tau_{\mathcal{D}}>(n-1) T\right\} \\
& \leqslant \cdots \leqslant q(T)^{n}=e^{-n \log q(T)^{-1}}
\end{aligned}
$$

The result follows from the fact that $n \geqslant(t / T)-1$.

Note that this estimate implies immediately

$$
\mathbb{E}^{x_{0}}\left[\tau_{\mathcal{D}}\right]=\int_{0}^{\infty} \mathbb{P}^{x_{0}}\left\{\tau_{\mathcal{D}}>t\right\} \mathrm{d} t \leqslant \frac{T}{q(T) \log q(T)^{-1}} .
$$

Our general strategy will be to find the smallest possible $T$ such that $q(T)$ is of order 1 , i.e., independent of the parameters $\kappa, \sigma$ and $\gamma$. Then $T$ will give the order of magnitude of the first-exit time.

When deriving these estimates, we will often encounter the process

$$
X_{t}=\frac{2 \gamma}{\sigma^{2}} \frac{1}{t} \int_{0}^{t} Z_{s}^{2} \mathrm{~d} s-1 .
$$


Lemma 5.3. For any initial condition $Z_{0}$, we have

$$
X_{t}=X_{\infty}+\bar{X}_{t}
$$

where $X_{\infty}$ has zero expectation, $\sqrt{X_{\infty}+1}$ follows a standard gaussian distribution, and $\bar{X}_{t}$ converges to zero in probability. More precisely, for any $\varepsilon>0$,

$$
\mathbb{P}\left\{\left|\bar{X}_{t}\right|>\varepsilon\right\}=\mathcal{O}\left(\frac{2 \gamma \mathbb{E}\left[Z_{0}^{2}\right]+\sigma^{2}}{2 \gamma t \varepsilon}\right)
$$

Proof: Write $Z_{t}=Z_{t}^{0}+\mathrm{e}^{-\gamma t}\left(Z_{0}-Z_{t}^{0}\right)$, where $Z_{t}^{0}$ is a stationary gaussian process of variance $\sigma^{2} / 2 \gamma$, and substitute in (5.14). The limit $X_{\infty}$ is obtained by applying the ergodic theorem to the stationary part. The bound (5.16) is a consequence of Markov's inequality applied to the remaining part.

We start by characterising the time needed to go from -1 to a level $-\delta_{1}$, where $\delta_{1}$ is a fixed constant in $(0,1)$. For an interval $I \subset[-1,1]$, we set

$$
\mathcal{D}_{I}=(-1,1) \times I \times \mathbb{S}^{1} \quad \text { and } \quad \tau_{I}=\tau_{\mathcal{D}_{I}} .
$$

Proposition 5.4. There exist constants $\sigma_{1}, c_{1}$ and $\kappa_{1}\left(\delta_{1}\right)=\mathcal{O}\left(\delta_{1}\right)$ such that whenever $\sigma<\sigma_{1}$ and $\kappa<\kappa_{1}$,

$$
\mathbb{P}^{x_{0}}\left\{\tau_{\left[-1,-\delta_{1}\right)}>c_{1}\left(\frac{1}{\gamma} \vee \frac{T^{\star}}{\delta_{1}}\right)\right\} \leqslant \frac{3}{4}
$$

holds for all $x_{0} \in \mathcal{D}_{\left[-1,-\delta_{1}\right)}$.

Proof: For $t \leqslant \tau_{\left[-1,-\delta_{1}\right)}$, we have $-\bar{y}_{t} \geqslant \delta_{1}$ and thus

$$
F\left(Z_{t}, \bar{y}_{t}, \phi_{t}\right) \geqslant \frac{4 \kappa^{2} \gamma}{1+\gamma^{2}} Z_{t}^{2}\left[\delta_{1}-\mathcal{O}(\kappa)\right] \geqslant \frac{2 \kappa^{2} \gamma}{1+\gamma^{2}} \delta_{1} Z_{t}^{2}
$$

provided $\kappa \leqslant \kappa_{1}=\mathcal{O}\left(\delta_{1}\right)$. By the comparison principle for SDEs, we thus have

$$
\bar{y}_{t}-\bar{y}_{0} \geqslant \frac{(\kappa \sigma)^{2} \delta_{1}}{1+\gamma^{2}} t\left(X_{t}+1\right)-\frac{2 \kappa \sigma}{1+\gamma^{2}} Y_{t},
$$

where we have introduced the martingale

$$
Y_{t}=-\frac{1+\gamma^{2}}{2 \kappa \sigma} \int_{0}^{t} G\left(Z_{s}, \bar{y}_{s}, \phi_{s}\right) \mathrm{d} W_{s}
$$

whose variance of is bounded above by a constant times $t$. Now we have, for any $T>0$,

$$
\begin{aligned}
\mathbb{P}^{x_{0}}\left\{\tau_{\left[-1,-\delta_{1}\right)}>T\right\} & \leqslant \mathbb{P}^{x_{0}}\left\{\bar{y}_{T}<-\delta_{1}\right\} \\
& \leqslant \mathbb{P}^{x_{0}}\left\{X_{T}<-K\right\}+\mathbb{P}^{x_{0}}\left\{Y_{T} \geqslant \frac{\kappa \sigma}{2} \delta_{1} T(1-K)+\frac{1+\gamma^{2}}{2 \kappa \sigma}\left(\delta_{1}+\bar{y}_{0}\right)\right\} .
\end{aligned}
$$

Lemma 5.3 implies that

$$
\begin{aligned}
\mathbb{P}^{x_{0}}\left\{X_{T}<-K\right\} & \leqslant \mathbb{P}^{x_{0}}\left\{X_{\infty}<-K+\sigma^{2}\right\}+\mathbb{P}^{x_{0}}\left\{\bar{X}_{T}<-\sigma^{2}\right\} \\
& =\Phi\left(\sqrt{1-K+\sigma^{2}}\right)-\Phi\left(-\sqrt{1-K+\sigma^{2}}\right)+\mathcal{O}\left(\frac{2 \gamma+\sigma^{2}}{2 \gamma \sigma^{2} T}\right)
\end{aligned}
$$


where $\Phi(x)=(2 \pi)^{-1 / 2} \int_{-\infty}^{x} \mathrm{e}^{-u^{2} / 2} \mathrm{~d} u$ denotes the distribution function of the standard normal law. Taking $K$ sufficiently close to 1 ( $K=15 / 16$ will do) and $\gamma T$ and $\sigma^{2} T$ sufficiently large allows to make this probability smaller than $1 / 4$. On the other hand, taking $\delta_{1} T$ larger than $2 T^{\star} /(1-K)$ allows us to bound the second term on the right-hand side of (5.22) by $\mathbb{P}^{x_{0}}\left\{Y_{T} \geqslant 0\right\}=1 / 2$.

It follows immediately from Lemma 5.2 that $\mathbb{P}^{x_{0}}\left\{\tau_{\left[-1,-\delta_{1}\right)}>t\right\}$ decreases exponentially fast with rate $\gamma \wedge \delta_{1} / T^{\star}$, and that

$$
\mathbb{E}^{x_{0}}\left[\tau_{\left[-1,-\delta_{1}\right)}\right]=\mathcal{O}\left(\frac{1}{\gamma} \vee \frac{T^{\star}}{\delta_{1}}\right)
$$

We now turn to estimating the time needed for $\bar{y}_{t}$ to reach a neighbourhood of zero. More precisely, we shall estimate

$$
\mathbb{P}^{x_{0}}\left\{\tau_{\left(-1+\delta_{2},-c^{\star} \kappa\right)}>t\right\}=\mathbb{P}^{x_{0}}\left\{-1+\delta_{2}<\bar{y}_{s}<-c^{\star} \kappa,\left|Z_{s}\right|<1 \forall s \leqslant t\right\},
$$

where the constant $c^{\star}$, which is related to error terms in (5.3), will be defined below. Let us start by considering, as a slight simplification of (5.2), the linear equation

$$
\mathrm{d} y_{t}^{0}=-\frac{4 \kappa^{2} \gamma}{1+\gamma^{2}} y_{t}^{0} \mathrm{~d} t+G\left(Z_{t}, \bar{y}_{t}, \phi_{t}\right) \mathrm{d} W_{t},
$$

with initial condition $y_{0}^{0}=\bar{y}_{0}$. Let

$$
\alpha(t)=\frac{4 \kappa^{2} \gamma}{1+\gamma^{2}} \int_{0}^{t} Z_{s}^{2} \mathrm{~d} s=\frac{2 t}{T^{\star}}\left(X_{t}+1\right) .
$$

Then Itô's formula shows that $y_{t}^{0}$ can be represented as

$$
y_{t}^{0}=\bar{y}_{0} \mathrm{e}^{-\alpha(t)}+\mathrm{e}^{-\alpha(t)} \int_{0}^{t} \mathrm{e}^{\alpha(s)} G\left(Z_{s}, \bar{y}_{s}, \phi_{s}\right) \mathrm{d} W_{s} .
$$

Lemma 5.5. Let $\tau_{0}=\inf \left\{t>0: y_{t}^{0}\right\}$ be the first time $y_{t}^{0}$ reaches 0 . Then

$$
\mathbb{P}\left\{\tau_{0}>t\right\} \leqslant \frac{2\left|\bar{y}_{0}\right|}{\sqrt{2 \pi v(t)}},
$$

where

$$
v(t)=\int_{0}^{t} \mathbb{E}\left[\mathrm{e}^{2 \alpha(s)} G\left(Z_{s}, \bar{y}_{s}, \phi_{s}\right)^{2}\right] \mathrm{d} s .
$$

Proof: When $\bar{y}_{0}=0$, the processes $y_{t}^{0}$ and $-y_{t}^{0}$ have the same distribution. Thus by the strong Markov property, we can apply André's reflection principle, yielding, for $\bar{y}_{0}>0$,

$$
\mathbb{P}\left\{\tau_{0}<t\right\}=2 \mathbb{P}\left\{y_{t}^{0} \leqslant 0\right\}
$$

and thus

$$
\mathbb{P}\left\{\tau_{0}>t\right\}=2 \mathbb{P}\left\{y_{t}^{0}>0\right\}-1 .
$$

Let $Y_{t}=\int_{0}^{t} \mathrm{e}^{\alpha(s)} G\left(Z_{s}, \bar{y}_{s}, \phi_{s}\right) \mathrm{d} W_{s}$. Being a stochastic integral, $Y_{t}$ is a Gaussian random variable, of expectation 0 , while Itô's formula shows that its variance is $v(t)$. Now observe that since $\mathrm{e}^{\alpha(t)}$ is a positive random variable, we have

$$
\begin{aligned}
\mathbb{P}\left\{y_{t}^{0}>0\right\} & =\mathbb{P}\left\{\bar{y}_{0}+Y_{t}>0\right\}=\mathbb{P}\left\{Y_{t}>-\bar{y}_{0}\right\} \\
& =\Phi\left(\frac{\bar{y}_{0}}{\sqrt{v(t)}}\right) \leqslant \frac{1}{2}+\frac{\bar{y}_{0}}{\sqrt{2 \pi v(t)}} .
\end{aligned}
$$

Inserting this in (5.32) yields the result. 
Before applying this to (5.2), we need another technical lemma, allowing to control the behaviour of $\phi_{t}$.

Lemma 5.6. Fix $\delta>0$ and an initial condition $x_{0}=\left(Z_{0}, \bar{y}_{0}, \phi_{0}\right) \in \mathcal{D}_{(-1+\delta, 1-\delta)}$. For any $\phi^{\star}>\phi_{0}$ and $\kappa<\delta / 2$, we have

$$
\tau_{\phi^{\star}}:=\inf \left\{t>0: \phi_{t}=\phi^{\star}\right\} \in\left[\frac{\phi^{\star}-\phi_{0}}{1+2 \kappa / \delta}, \frac{\phi^{\star}-\phi_{0}}{1-2 \kappa / \delta}\right] \cup\left[\tau_{(-1+\delta, 1-\delta)}, \infty\right] .
$$

PROOF: Either $\tau_{\phi^{\star}} \geqslant \tau_{(-1+\delta, 1-\delta)}$ and we are done, or $\tau_{\phi^{\star}}<\tau_{(-1+\delta, 1-\delta)}$. In the latter case, (5.1) implies that $[1-2 \kappa / \delta] \mathrm{d} t \leqslant \mathrm{~d} \phi_{t} \leqslant[1+2 \kappa / \delta] \mathrm{d} t$, and the result follows by integrating from 0 to $\tau_{\phi^{\star}}$.

Proposition 5.7. There exist constants $c^{\star}, c_{2}$ and $\kappa_{2}=\mathcal{O}\left(\delta_{2}\right)$ such that whenever $\kappa<\kappa_{2}$,

$$
\mathbb{P}^{x_{0}}\left\{\tau_{\left(-1+\delta_{2},-c^{\star} \kappa\right)}>t\right\} \leqslant \frac{c_{2}\left|\bar{y}_{0}\right|}{\sqrt{\delta_{2}}} \exp \left\{-\frac{2 t}{T^{\star}}\right\}
$$

holds for all $x_{0} \in \mathcal{D}_{\left(-1+\delta_{2},-c^{\star} \kappa\right)}$ and all $t \geqslant T^{\star}$.

Proof: For $t \leqslant \tau_{\left(-1+\delta_{2}, 1-\delta_{2}\right)}$, we have $\left|Z_{t}\right|<1$ and thus

$$
F\left(Z_{t}, \bar{y}_{t}, \phi_{t}\right) \geqslant-\frac{4 \kappa^{2} \gamma}{1+\gamma^{2}} Z_{t}^{2}\left[\bar{y}+c^{\star} \kappa\right]
$$

for some $c^{\star}>0$. By the comparison principle for SDEs, it follows that $\bar{y}_{t}+c^{\star} \kappa \geqslant y_{t}^{0}$, where $y_{t}^{0}$ obeys the SDE (5.26). We can thus apply Lemma 5.5, and the proof amounts to finding an exponentially growing lower bound for the variance $v(t)$ defined in (5.30).

Since for $t \leqslant \tau_{\left(-1+\delta_{2},-c^{\star} \kappa\right)}$, we have $1-\bar{y}_{t}^{2} \geqslant 2 \delta_{2}-\delta_{2}^{2} \geqslant \delta_{2}$, if follows that

$$
G\left(Z_{t}, \bar{y}_{t}, \phi_{t}\right)^{2} \geqslant \frac{4 \delta_{2}}{T^{\star}} \cos ^{2}\left(\phi_{t}+\theta\right)
$$

for these times. We can thus write, using Jensen's inequality,

$$
\begin{aligned}
\frac{T^{\star}}{4 \delta_{2}} v(t) & =\frac{T^{\star}}{4 \delta_{2}} \int_{0}^{t} \mathbb{E}\left[\mathrm{e}^{2 \alpha(s)} G\left(Z_{s}, \bar{y}_{s}, \phi_{s}\right)^{2}\right] \mathrm{d} s \\
& \geqslant \frac{1}{2} \int_{0}^{t} 1_{\left\{\cos ^{2}\left(\phi_{s}+\theta\right) \geqslant 1 / 2\right\}} \mathbb{E}\left[\mathrm{e}^{2 \alpha(s)}\right] \mathrm{d} s \\
& \geqslant \frac{1}{2} \int_{0}^{t} 1_{\left\{\cos ^{2}\left(\phi_{s}+\theta\right) \geqslant 1 / 2\right\}} \mathrm{e}^{2 \mathbb{E}[\alpha(s)]} \mathrm{d} s \\
& \geqslant \frac{1}{2} \int_{0}^{t} 1_{\left\{\cos ^{2}\left(\phi_{s}+\theta\right) \geqslant 1 / 2\right\}} \mathrm{e}^{4 s / T^{\star}} \mathrm{d} s .
\end{aligned}
$$

Lemma 5.6 implies that (taking $\left.\kappa \leqslant \delta_{2} / 4\right)$ during any time interval of length $\pi /\left(1-2 \kappa / \delta_{2}\right)$, $\cos ^{2}\left(\phi_{s}+\theta\right)$ stays larger than $1 / 2$ for a time span of length $\pi / 2\left(1+2 \kappa / \delta_{2}\right)$ at least. This implies that

$$
\begin{aligned}
\int_{k \pi /\left(1-2 \kappa / \delta_{2}\right)}^{(k+1) \pi /\left(1-2 \kappa / \delta_{2}\right)} 1_{\left\{\cos ^{2} \phi_{s} \geqslant 1 / 2\right\}} \mathrm{e}^{4 s / T^{\star}} \mathrm{d} s & \geqslant \frac{T^{\star}}{4} \mathrm{e}^{4 k \pi / T^{\star}\left(1-2 \kappa / \delta_{2}\right)}\left[\mathrm{e}^{2 \pi / T^{\star}\left(1+2 \kappa / \delta_{2}\right)}-1\right] \\
& \geqslant \frac{\pi}{2\left(1+2 \kappa / \delta_{2}\right)} \mathrm{e}^{4 k \pi / T^{\star}\left(1-2 \kappa / \delta_{2}\right)}
\end{aligned}
$$

This allows the variance $v(t)$ to be bounded below by a geometric series, whose sum is of order $\delta_{2}\left(\mathrm{e}^{4 t / T^{\star}}-1\right)$. The result thus follows from Lemma 5.5 . 
Finally, we consider the problem of $\bar{y}_{t}$ reaching any level in $(-1,1)$.

Proposition 5.8. For any $\delta_{3}>0$, there exist constants $c_{3}$, $h$ and $\kappa_{3}=\mathcal{O}\left(\delta_{3}\right)$ such that whenever $\kappa<\kappa_{3}$,

$$
\mathbb{P}^{x_{0}}\left\{\tau_{\left(-1+\delta_{3}, 1-\delta_{3}\right)}>\frac{c_{3}}{\delta_{3}}\left(\frac{1}{\gamma} \vee T^{\star}\right)\right\} \leqslant \Phi\left(\frac{1-\delta_{3}}{h \sqrt{\delta_{3}}}\right)
$$

holds for all $x_{0} \in \mathcal{D}_{\left(-1+\delta_{3}, 1-\delta_{3}\right)}$.

Proof: As in the proof of Proposition 5.7, we can bound $\bar{y}_{t}$ below by $y_{t}^{0}-\kappa$, where $y_{t}^{0}$ is the solution of the linear equation (5.26). Now for any $T, L>0$, we can write

$$
\begin{aligned}
\mathbb{P}^{x_{0}}\left\{\sup _{0 \leqslant t \leqslant T} y_{t}^{0}<L\right\} \leqslant & \mathbb{P}^{x_{0}}\left\{\inf _{T / 2 \leqslant t \leqslant T} \frac{\alpha(t)}{t} \geqslant \frac{2}{T^{\star}}\right\} \\
& +\mathbb{P}^{x_{0}}\left\{\sup _{T / 2 \leqslant t \leqslant T} y_{t}^{0}<L, \exists t \in[T / 2, T]: \alpha(t)<\frac{2 t}{T^{\star}}\right\} .
\end{aligned}
$$

Using the process $X_{t}$ introduced in (5.14), the first term on the right-hand side can be rewritten as

$$
\mathbb{P}^{x_{0}}\left\{\inf _{T / 2 \leqslant t \leqslant T} X_{t} \geqslant 0\right\}
$$

which decreases exponentially fast, like $\mathrm{e}^{-\gamma T}$. Choosing $\gamma T \geqslant \mathcal{O}\left(1 / \delta_{3}\right)$ makes this term of order $\mathrm{e}^{-1 / \delta_{3}}$. Let again $Y_{t}$ denote the stochastic integral $\int_{0}^{t} \mathrm{e}^{\alpha(s)} G\left(Z_{s}, \bar{y}_{s}, \phi_{s}\right) \mathrm{d} W_{s}$. The second term on the right-hand side of (5.41) can be bounded by

$$
\begin{aligned}
& \mathbb{P}^{x_{0}}\left\{\sup _{T / 2 \leqslant t \leqslant T} \mathrm{e}^{-\alpha(t)}\left(\bar{y}_{0}+Y_{t}\right)<L, \exists t \in[T / 2, T]: \alpha(t)<\frac{2 t}{T^{\star}}\right\} \\
& \leqslant \mathbb{P}^{x_{0}}\left\{\exists t \in[T / 2, T]: Y_{t}<L \mathrm{e}^{2 t / T^{\star}}-\bar{y}_{0}\right\} \\
& \quad \leqslant \sup _{t \in[T / 2, T]} \Phi\left(\frac{L \mathrm{e}^{2 t / T^{\star}}-\bar{y}_{0}}{\sqrt{v(t)}}\right),
\end{aligned}
$$

where $v(t)$ can be estimated with the help of (5.38). Taking $T$ sufficiently large that $\mathrm{e}^{-2 T / T^{\star}} \leqslant \delta_{3} / 2$ allows to bound $v(T / 2)$ below by $\left(\delta_{3} / 2\right) \mathrm{e}^{2 T / T^{\star}}$. The argument of $\Phi$ can thus be bounded by a constant times $1 / \sqrt{\delta_{3}}$, while $L=1-\delta_{3}$. This yields the result.

A partially matching lower bound is given by the following estimate.

Proposition 5.9. There exists a constant $c_{4}$ such that for any $L \in(0,1)$ and any initial condition $x_{0}=\left(Z_{0}, \bar{y}_{0}, \phi_{0}\right)$ with $\bar{y}_{0}>-L$,

$$
\mathbb{P}^{x_{0}}\left\{\inf _{0 \leqslant t \leqslant T^{\star}} \bar{y}_{t}<-L\right\} \leqslant \exp \left\{-c_{4}\left[L+\bar{y}_{0}-c^{\star} \kappa\right]^{2}\right\} .
$$

Proof: Using the same notations as in the previous proof, and the fact that $\alpha(t) \geqslant 0$, we can bound the probability by

$$
\mathbb{P}^{x_{0}}\left\{\sup _{0 \leqslant t \leqslant T^{\star}}\left(-Y_{t}\right)>L+\bar{y}_{0}-c^{\star} \kappa\right\} .
$$

Since $Y_{t}$ is a martingale, a standard argument based on Doob's submartingale inequality applied to $\mathrm{e}^{\eta Y_{t}}$ (cf. [BG03, Lemma 3.3]) allows to bound this probability by the exponential of $-c_{4}\left(L+\bar{y}_{0}-c^{\star} \kappa\right)^{2} / v\left(T^{\star}\right)$. The variance $v\left(T^{\star}\right)$ can be bounded above by a constant. 


\subsection{Renewal equations}

We now have to patch together the different estimates obtained in the previous section in order to bound the expected transition time between general domains in phase space. We shall do this by using renewal equations, that we will solve using Laplace transforms. Recall that the Laplace transform of a random variable $\tau$ taking values in $\mathbb{R}_{+}$is given by

$$
\mathbb{E}\left[\mathrm{e}^{\lambda \tau}\right]=\int_{0}^{\infty} \mathrm{e}^{\lambda t} \frac{\partial}{\partial t} \mathbb{P}\{\tau \leqslant t\} \mathrm{d} t=1+\lambda \int_{0}^{\infty} \mathrm{e}^{\lambda t} \mathbb{P}\{\tau>t\} \mathrm{d} t
$$

provided $\lambda$ is such that $\lim _{t \rightarrow \infty} \mathrm{e}^{\lambda t} \mathbb{P}\{\tau>t\}=0$. If $\tau$ takes values in $\mathbb{R}+\cup\{\infty\}$, we can also define

$$
\mathbb{E}\left[\mathrm{e}^{\lambda \tau} 1_{\{\tau<\infty\}}\right]=\mathbb{P}\{\tau<\infty\}+\lambda \int_{0}^{\infty} \mathrm{e}^{\lambda t} \mathbb{P}\{t<\tau<\infty\} \mathrm{d} t,
$$

provided $\lambda$ is such that $\lim _{t \rightarrow \infty} \mathrm{e}^{\lambda t} \mathbb{P}\{t<\tau<\infty\}=0$.

It is instructive to consider first the case of a one-dimensional process $\left\{y_{t}\right\}_{t \geqslant 0}$, solution to a one-dimensional autonomous stochastic differential equation. We assume that $y_{t} \in$ $[-1,1]$ for all $t$, and introduce levels $-1<h_{1}<h_{2}<h_{3}<1$ (Figure 33a). We set $\mathcal{D}_{1}=\left[-1, h_{2}\right], \mathcal{D}_{2}=\left[h_{1}, h_{3}\right]$ and introduce first-exit times $\tau_{1}=\tau_{\mathcal{D}_{1}}, \tau_{2}=\tau_{\mathcal{D}_{2}}, \tau=\tau_{\mathcal{D}_{1} \cup \mathcal{D}_{2}}$ and

$$
\tau_{\searrow}=\inf \left\{t>0: y_{t}<h_{1}\right\} .
$$

The following result allows to express the expectation of $\tau$ using informations on $\tau_{1}, \tau_{2}$ and $\tau_{\searrow}$.

Proposition 5.10. Assume there exists an $\varepsilon>0$ such that the Laplace transforms $\mathbb{E}^{h_{1}}\left[\mathrm{e}^{\lambda \tau_{1}}\right]$ and $\mathbb{E}^{h_{2}}\left[\mathrm{e}^{\lambda \tau_{2}}\right]$ are finite for all $\lambda<\varepsilon$. Then

$$
\mathbb{E}^{h_{1}}[\tau]=\frac{\mathbb{E}^{h_{1}}\left[\tau_{1}\right]+\mathbb{E}^{h_{2}}\left[\tau_{2}\right]}{\mathbb{P}^{h_{2}}\left\{\tau_{2}<\tau_{\searrow}\right\}} .
$$

Proof: Since $\tau>\tau_{1}$ for all initial conditions in $\mathcal{D}_{1}$, we have, by the strong Markov property and time homogeneity, the renewal equation

$$
\begin{aligned}
\mathbb{P}^{h_{1}}\{\tau>t\} & =\mathbb{P}^{h_{1}}\left\{\tau_{1}>t\right\}+\mathbb{E}^{h_{1}}\left[1_{\left\{\tau_{1} \leqslant t\right\}} \mathbb{P}^{h_{2}, \tau_{1}}\{\tau>t\}\right] \\
& =\mathbb{P}^{h_{1}}\left\{\tau_{1}>t\right\}+\int_{0}^{t} \frac{\partial}{\partial s} \mathbb{P}^{h_{1}}\left\{\tau_{1} \leqslant s\right\} \mathbb{P}^{h_{2}}\{\tau>t-s\} \mathrm{d} s
\end{aligned}
$$

Taking the Laplace transform of (5.50) and making the change of variables $t-s=u$ shows that

$$
\begin{aligned}
\mathbb{E}^{h_{1}}\left[\mathrm{e}^{\lambda \tau}\right] & =\mathbb{E}^{h_{1}}\left[\mathrm{e}^{\lambda \tau_{1}}\right]+\lambda \int_{0}^{\infty} \mathrm{e}^{\lambda s} \frac{\partial}{\partial s} \mathbb{P}^{h_{1}}\left\{\tau_{1} \leqslant s\right\} \mathrm{d} s \int_{0}^{\infty} \mathrm{e}^{\lambda u} \mathbb{P}^{h_{2}}\{\tau>u\} \mathrm{d} u \\
& =\mathbb{E}^{h_{1}}\left[\mathrm{e}^{\lambda \tau_{1}}\right]+\mathbb{E}^{h_{1}}\left[\mathrm{e}^{\lambda \tau_{1}}\right]\left(\mathbb{E}^{h_{2}}\left[\mathrm{e}^{\lambda \tau}\right]-1\right) \\
& =\mathbb{E}^{h_{1}}\left[\mathrm{e}^{\lambda \tau_{1}}\right] \mathbb{E}^{h_{2}}\left[\mathrm{e}^{\lambda \tau}\right] .
\end{aligned}
$$

Now since for initial conditions in $\mathcal{D}_{2}, \tau_{2}=\tau \wedge \tau_{\searrow}$, we also have the renewal equation

$$
\mathbb{P}^{h_{2}}\{\tau>t\}=\mathbb{P}^{h_{2}}\left\{\tau_{2}>t\right\}+\mathbb{E}^{h_{2}}\left[1_{\left\{\tau_{\searrow} \leqslant t \wedge \tau_{2}\right\}} \mathbb{P}^{h_{1}, \tau} \searrow\{\tau>t\}\right]
$$


(a)

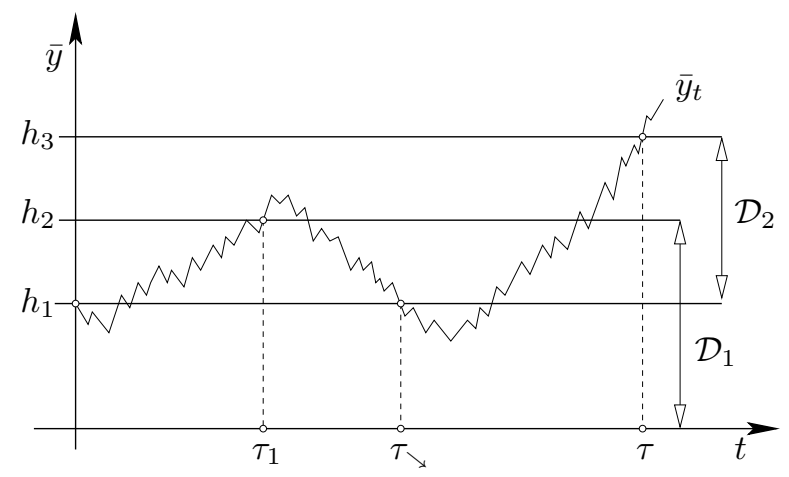

(b)

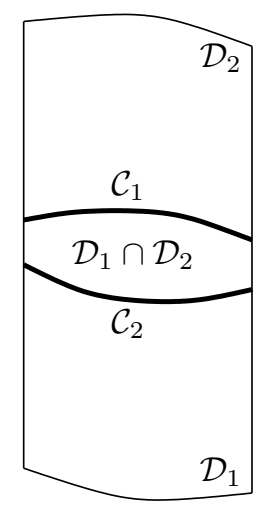

Figure 3. (a) The setting of Proposition 5.10, The first-exit time $\tau_{2}$ from $\mathcal{D}_{2}$ being the minimum $\tau_{\searrow} \wedge \tau$, the distribution of $\tau$ can be related to the distributions of $\tau_{1}, \tau_{2}$ and $\tau_{\searrow}$.

(b) The setting of Proposition 5.12 .

The condition $\tau_{\searrow} \leqslant t \wedge \tau_{2}$ can be rewritten as $\tau_{\searrow}^{\#} \leqslant t$, where $\tau_{\searrow}^{\#} \in \mathbb{R}_{+} \cup\{\infty\}$ denotes the first time the process $y_{t}$, killed upon reaching $h_{3}$, reaches $h_{1}$ (we set $\tau_{\searrow}^{\#}=\infty$ in case this never happens). Taking the Laplace transform, we get

$$
\mathbb{E}^{h_{2}}\left[\mathrm{e}^{\lambda \tau}\right]=\mathbb{E}^{h_{2}}\left[\mathrm{e}^{\lambda \tau_{2}}\right]+\mathbb{E}^{h_{2}}\left[\mathrm{e}^{\lambda \tau^{\#}} 1_{\left\{\tau_{\searrow}^{\#}<\infty\right\}}\right]\left(\mathbb{E}^{h_{1}}\left[\mathrm{e}^{\lambda \tau}\right]-1\right)
$$

Combining (5.51) with (5.53), we obtain

$$
\mathbb{E}^{h_{1}}\left[\mathrm{e}^{\lambda \tau}\right]=\frac{\mathbb{E}^{h_{1}}\left[\mathrm{e}^{\lambda \tau_{1}}\right]\left(\mathbb{E}^{h_{2}}\left[\mathrm{e}^{\lambda \tau_{2}}\right]-\mathbb{E}^{h_{2}}\left[\mathrm{e}^{\lambda \tau_{\searrow}^{\#}} 1_{\left\{\tau_{\searrow}<\infty\right\}}\right]\right)}{1-\mathbb{E}^{h_{1}}\left[\mathrm{e}^{\lambda \tau_{1}}\right] \mathbb{E}^{h_{2}}\left[\mathrm{e}^{\lambda \tau_{\searrow}^{\#}} 1_{\left\{\tau_{\searrow}^{\#}<\infty\right\}}\right]}
$$

The result then follows by taking the derivative of this relation with respect to $\lambda$, evaluating it in $\lambda=0$, and using the fact that $\mathbb{P}^{h_{2}}\left\{\tau_{\searrow}^{\#}<\infty\right\}=\mathbb{P}^{h_{2}}\left\{\tau_{\searrow}<\tau_{2}\right\}$.

Remark 5.11. Relation (5.54) shows that we do in fact control the Laplace transform, and thus the distribution of $\tau$. Since

$$
\mathbb{P}\{\tau>t\}=\mathbb{P}\left\{\mathrm{e}^{\lambda \tau}>\mathrm{e}^{\lambda t}\right\} \leqslant \mathrm{e}^{-\lambda t} \mathbb{E}\left[\mathrm{e}^{\lambda \tau}\right]
$$

for all $\lambda$ such that $\mathbb{E}\left[\mathrm{e}^{\lambda \tau}\right]$ is bounded, we see that the tails of the distribution of $\tau$ decay exponentially fast, with a rate controlled by the domain of the Laplace transform. This domain depends on the rate of exponential decay of $\tau_{1}$ and $\tau_{2}$ and on the denominator in (5.54).

Relation (5.49) has a slightly asymmetric form, due to the fact that in the situation considered, we always have $\tau_{1}<\tau$. It can however easily be extended to situations where this relation does not hold. We will consider the still more general situation of a timehomogeneous SDE in $\mathbb{R}^{n}$. Let $\mathcal{D}_{1}$ and $\mathcal{D}_{2}$ be two open sets in $\mathbb{R}^{n}$, and let $\tau_{1}, \tau_{2}$ and $\tau$ denote the first-exit times from $\mathcal{D}_{1}, \mathcal{D}_{2}$ and $\mathcal{D}_{1} \cup \mathcal{D}_{2}$ respectively. Finally let $\mathcal{C}_{1}=\partial \mathcal{D}_{1} \cap \mathcal{D}_{2}$ and $\mathcal{C}_{2}=\partial \mathcal{D}_{2} \cap \mathcal{D}_{1}$ (Figure $3 \mathrm{~b}$ ). 
Proposition 5.12. Assume there exists an $\varepsilon>0$ such that the Laplace transforms of $\tau_{1}$ and $\tau_{2}$ are finite for all $\lambda<\varepsilon$. Then

$$
\sup _{x_{0} \in \mathcal{D}_{1} \cup \mathcal{D}_{2}} \mathbb{E}^{x_{0}}[\tau] \leqslant \frac{2\left(\sup _{x \in \mathcal{D}_{1}} \mathbb{E}^{x}\left[\tau_{1}\right]+\sup _{y \in \mathcal{D}_{2}} \mathbb{E}^{y}\left[\tau_{2}\right]\right)}{1-\sup _{x \in \mathcal{C}_{2}} \mathbb{P}^{x}\left\{x_{\tau_{1}} \in \mathcal{C}_{1}\right\} \sup _{y \in \mathcal{C}_{1}} \mathbb{P}^{y}\left\{x_{\tau_{2}} \in \mathcal{C}_{2}\right\}} .
$$

Proof: Let $\tau_{1}^{\#}$ denote the first-exit time from $\mathcal{D}_{1}$ of the process killed upon hitting $\partial \mathcal{D}_{1} \backslash \mathcal{D}_{2}$, i.e., we set $\tau_{1}^{\#}=\infty$ in case $x_{t}$ hits $\partial \mathcal{D}_{1} \backslash \mathcal{D}_{2}$ before $\mathcal{C}_{1}$. Then we have, for initial conditions $x \in \mathcal{D}_{1}$,

$$
\mathbb{P}^{x}\{\tau>t\}=\mathbb{P}^{x}\left\{\tau_{1}>t\right\}+\mathbb{E}^{x}\left[1_{\left\{\tau_{1}^{\#}<t\right\}} \mathbb{P}^{\left.x_{\tau_{1}^{*}}^{\#}\left\{\tau>t-\tau_{1}^{\#}\right\}\right]} .\right.
$$

Taking the Laplace transform, and using the change of variables $t=\tau_{1}^{\#}+s$, we get

$$
\mathbb{E}^{x}\left[\mathrm{e}^{\lambda \tau}\right]=\mathbb{E}^{x}\left[\mathrm{e}^{\lambda \tau_{1}}\right]+\lambda \int_{0}^{\infty} \mathrm{e}^{\lambda s} \mathbb{E}^{x}\left[\mathrm{e}^{\lambda \tau_{1}^{\#}} 1_{\left\{\tau_{1}^{\#}<\infty\right\}} \mathbb{P}^{x_{\tau_{1}^{*}}^{\#}}\{\tau>s\}\right] \mathrm{d} s .
$$

Evaluating the derivative in $\lambda=0$ yields the bound

$$
\mathbb{E}^{x}[\tau] \leqslant \mathbb{E}^{x}\left[\tau_{1}\right]+\sup _{y \in \mathcal{C}_{1}} \mathbb{E}^{y}[\tau] \mathbb{P}^{x}\left\{\tau_{1}^{\#}<\infty\right\}
$$

A similar relation holds for initial conditions $y \in \mathcal{D}_{2}$. Combining both relations after taking the supremum over $x \in \mathcal{C}_{2}$ and $y \in \mathcal{C}_{1}$, we get

$$
\sup _{x \in \mathcal{C}_{2}} \mathbb{E}^{x}[\tau] \leqslant \frac{\sup _{x \in \mathcal{C}_{2}} \mathbb{E}^{x}\left[\tau_{1}\right]+\sup _{y \in \mathcal{C}_{1}} \mathbb{E}^{y}\left[\tau_{2}\right] \sup _{x \in \mathcal{C}_{2}} \mathbb{P}^{x}\left\{\tau_{1}^{\#}<\infty\right\}}{1-\sup _{x \in \mathcal{C}_{2}} \mathbb{P}^{x}\left\{\tau_{1}^{\#}<\infty\right\} \sup _{y \in \mathcal{C}_{1}} \mathbb{P}^{y}\left\{\tau_{2}^{\#}<\infty\right\}},
$$

and a similar relation for initial conditions in $\mathcal{C}_{1}$. Using again (5.59) and its equivalent, but now for general $x_{0} \in \mathcal{D}_{1}$ and $y_{0} \in \mathcal{D}_{2}$, yields the result (after some simplifications the upper bound is not sharp).

\subsection{Proof of Theorem 2.7}

We can now proceed to the proof of Theorem 2.7, characterising the expectation of the first-passage time

$$
\tau(y)=\inf \left\{t>0: \bar{y}_{t}>y\right\}
$$

of $\bar{y}_{t}$ at any level $y \in[-1,1]$. Notice that we have (cf. (5.17))

$$
\tau_{[-1, y)}=\tau(y) \wedge \tau_{Z},
$$

where

$$
\tau_{Z}=\inf \left\{t>0:\left|Z_{t}\right|>1\right\}
$$

is typically very large for small $\sigma$. We start by bounding the expectation of $\tau_{[-1, y)}$.

Proposition 5.13. For any $x_{0}=\left(Z_{0}, \bar{y}_{0}, \phi_{0}\right)$ with $\left|Z_{0}\right| \leqslant 1$,

$$
\mathbb{E}^{x_{0}}\left[\tau_{[-1, y)}\right] \leqslant e_{1}(y)\left[\frac{1}{\gamma} \vee T^{\star}\right],
$$

where the function $e_{1}(y)$ is bounded for $y<1$. 
Proof: We start by setting $\mathcal{D}_{1}=\mathcal{D}_{\left[-1,-\delta_{1}\right)}$ and $\mathcal{D}_{2}=\mathcal{D}_{\left(-1+\delta_{2},-c^{\star} \kappa\right)}$. Then Proposition 5.12 yields a bound of the form

$$
\mathbb{E}^{x_{0}}\left[\tau_{\left[-1,-c^{\star} \kappa\right)}\right] \leqslant \frac{2\left(E_{1}+E_{2}\right)}{1-P_{1} P_{2}},
$$

where Proposition 5.4 shows

$$
E_{1}=\sup _{x \in \mathcal{D}_{\left[-1,-\delta_{1}\right)}} \mathbb{E}^{x}\left[\tau_{\left[-1,-\delta_{1}\right)}\right]=\mathcal{O}\left(\frac{1}{\gamma} \vee \frac{T^{\star}}{\delta_{1}}\right),
$$

and Proposition 5.7 yields

$$
E_{2}=\sup _{x \in \mathcal{D}_{\left(-1+\delta_{2},-c^{\star} \kappa\right)}} \mathbb{E}^{x}\left[\tau_{\left(-1+\delta_{2},-c^{\star} \kappa\right)}\right] \leqslant \frac{c_{2}}{\sqrt{\delta_{2}}} T^{\star} .
$$

We expect $P_{1}$ to be very close to 1 , so we only try to bound $P_{2}$, which is done as follows. Let $\tau_{\searrow}$ and $\tau_{\nearrow}$ denote the first-hitting times of the levels $-1+\delta_{2}$ and $-c^{\star} \kappa$ respectively. Then

$$
\begin{aligned}
P_{2} & =\sup _{x: \bar{y}=-\delta_{1}} \mathbb{P}^{x}\left\{\bar{y}_{\tau_{\left[-1,-\delta_{1}\right)}}=-1+\delta_{2}\right\} \\
& =\sup _{x: \bar{y}=-\delta_{1}} \mathbb{P}^{x}\left\{\tau_{\searrow}<\tau_{\nearrow}\right\} \\
& \leqslant \sup _{x: \bar{y}=-\delta_{1}}\left[\mathbb{P}^{x}\left\{\tau_{\searrow}<T^{\star}\right\}+\mathbb{P}^{x}\left\{\tau_{\nearrow}>T^{\star}\right\}\right] .
\end{aligned}
$$

Proposition $\left[5.9\right.$ shows that $\mathbb{P}^{x}\left\{\tau_{\searrow}<T^{\star}\right\}$ is of order $\mathrm{e}^{-c_{4}\left(1-\delta_{2}-2 c^{\star} \kappa\right)^{2}}$, while Proposition 5.7 shows that $\mathbb{P}^{x}\left\{\tau_{\nearrow}>T^{\star}\right\}$ is of order $\left|\delta_{1}\right| / \sqrt{\delta_{2}}$. Thus choosing, for instance, $\delta_{2}=1 / 2$, and $\delta_{1}$ small enough makes $P_{2}$ bounded away from one. This proves (5.64) for all $y<-c^{\star} \kappa$. We now repeat the procedure, going first up to level $1 / 2$, and finally to level $1-\delta_{3}$. The $\delta_{i}$ 's can be chosen each time in such a way that $\mathbb{P}\left\{\tau_{\searrow}<\tau_{\nearrow}\right\}$ is bounded away from 1.

Proof of Theorem 2.7. We will use the fact that the Ornstein-Uhlenbeck process $Z_{t}$ reaches 0 in a time of order $1 / \gamma$, and needs an exponentially long time to go from 0 to 1 . In order to apply Proposition 5.12, we introduce sets

$$
\begin{aligned}
& \mathcal{D}_{1}=(-1,1) \times[-1, y) \times \mathbb{S}^{1}=\mathcal{D}_{[-1, y)} \\
& \mathcal{D}_{2}=\mathbb{R}^{*} \times(-1, y) \times \mathbb{S}^{1} .
\end{aligned}
$$

Then the first-exit time from $\mathcal{D}_{2}$ equals $\tau_{0} \wedge \tau(y)$, where $\tau_{0}$ denotes the first time $Z_{t}$ hits 0 . We have $\mathcal{C}_{1}=\partial \mathcal{D}_{1} \cap \mathcal{D}_{2}=\{-1,1\} \times(-1, y) \times \mathbb{S}^{1}$ and $\mathcal{C}_{2}=\partial \mathcal{D}_{2} \cap \mathcal{D}_{1}=\{0\} \times(-1, y) \times \mathbb{S}^{1}$. Thus (5.60) yields

$$
\sup _{x \in \mathcal{C}_{2}} \mathbb{E}^{x}[\tau(y)] \leqslant \frac{\sup _{x \in \mathcal{C}_{2}} \mathbb{E}^{x}\left[\tau_{[-1, y)}\right]+\sup _{y \in \mathcal{C}_{1}} \mathbb{E}^{y}\left[\tau_{0}\right] \sup _{x \in \mathcal{C}_{2}} \mathbb{P}^{x}\left\{\tau_{Z} \leqslant \tau(y)\right\}}{1-\sup _{x \in \mathcal{C}_{2}} \mathbb{P}^{x}\left\{\tau_{Z} \leqslant \tau(y)\right\} \sup _{y \in \mathcal{C}_{1}} \mathbb{P}^{y}\left\{\tau_{0} \leqslant \tau(y)\right\}} .
$$

Using the reflection principle, one obtains $\mathbb{E}^{y}\left[\tau_{0}\right]=\mathcal{O}(1 / \gamma)$. Furthermore, we can write for any $K>0$

$$
\mathbb{P}^{x}\left\{\tau_{Z} \leqslant \tau(y)\right\} \leqslant \mathbb{P}^{x}\left\{\tau_{Z} \leqslant K\right\}+\mathbb{P}^{x}\left\{\tau(y) \wedge \tau_{Z}>K\right\}
$$


We choose $K=N\left(\gamma^{-1} \vee T^{\star}\right)$ for some $N>0$. Then on one hand, Markov's inequality, (5.62) and Proposition 5.13 yield

$$
\mathbb{P}^{x}\left\{\tau(y) \wedge \tau_{Z}>K\right\}=\mathbb{P}^{x}\left\{\tau_{[-1, y)}>K\right\} \leqslant \frac{\mathbb{E}^{x_{2}}\left[\tau_{[-1, y)}\right]}{K} \leqslant \frac{e_{1}(y)}{N} .
$$

On the other hand, a well-known property of the Ornstein-Uhlenbeck process is that $\mathbb{P}^{x}\left\{\tau_{Z} \leqslant K\right\}$ behaves like $(K / \sigma) \mathrm{e}^{-\gamma / \sigma^{2}}$ (see, for instance, [BG02, Proposition 3.3] or [BG04, Appendix]). Thus choosing for instance $N^{2}=e_{1}(y) \sigma \mathrm{e}^{\gamma / \sigma^{2}} /\left(\gamma^{-1} \vee T^{\star}\right)$ makes $\mathbb{P}^{x}\left\{\tau_{Z} \leqslant \tau(y)\right\}$ of order $\sqrt{e_{1}(y)\left(\gamma^{-1} \vee T^{\star}\right)} \sigma^{-1 / 2} \mathrm{e}^{-\gamma / 2 \sigma^{2}}$, which is negligible compared to the expectation of $\tau_{[-1, y)}$ and to 1 . This proves the result for initial conditions $x \in \mathcal{C}_{2}$.

In order to extend it to general initial conditions, it suffices to apply (5.59) and its twin relation, and to integrate over the initial distribution of $Z$.

\section{Acknowledgements}

This work has been supported by the French Ministry of Research, by way of the Action Concertée Incitative (ACI) Jeunes Chercheurs, Modélisation stochastique de systèmes hors équilibre.

\section{References}

[AJ07a] Stéphane Attal and Alain Joye, The Langevin equation for a quantum heat bath, J. Funct. Anal. 247 (2007), no. 2, 253-288.

[AJ07b] Weak coupling and continuous limits for repeated quantum interactions, J. Stat. Phys. 126 (2007), no. 6, 1241-1283.

[AP06] Stéphane Attal and Yan Pautrat, From repeated to continuous quantum interactions, Ann. Henri Poincaré 7 (2006), no. 1, 59-104.

[BG02] Nils Berglund and Barbara Gentz, Pathwise description of dynamic pitchfork bifurcations with additive noise, Probab. Theory Related Fields 122 (2002), no. 3, 341-388.

[BG03] — Geometric singular perturbation theory for stochastic differential equations, J. Differential Equations 191 (2003), 1-54.

[BG04] - On the noise-induced passage through an unstable periodic orbit I: Two-level model, J. Statist. Phys. 114 (2004), 1577-1618.

[BG06] _ Noise-induced phenomena in slow-fast dynamical systems. a sample-paths approach, Probability and its Applications, Springer-Verlag, London, 2006.

[BJM06] Laurent Bruneau, Alain Joye, and Marco Merkli, Asymptotics of repeated interaction quantum systems, J. Funct. Anal. 239 (2006), no. 1, 310-344.

[DV76] M. D. Donsker and S. R. S. Varadhan, On the principal eigenvalue of second-order elliptic differential operators, Comm. Pure Appl. Math. 29 (1976), no. 6, 595-621.

[EPRB99] J.-P. Eckmann, C.-A. Pillet, and L. Rey-Bellet, Non-equilibrium statistical mechanics of anharmonic chains coupled to two heat baths at different temperatures, Comm. Math. Phys. 201 (1999), no. 3, 657-697.

[FKM65] G. W. Ford, M. Kac, and P. Mazur, Statistical mechanics of assemblies of coupled oscillators, J. Mathematical Phys. 6 (1965), 504-515.

[FW04] Mark Freidlin and Matthias Weber, Random perturbations of dynamical systems and diffusion processes with conservation laws, Probab. Theory Related Fields 128 (2004), no. $3,441-466$. 
[Jen12] Jentzsch, Über Integralgleichungen mit positivem Kern, J. f. d. reine und angew. Math. 141 (1912), 235-244.

[LL58] L. D. Landau and E. M. Lifshitz, Quantum mechanics: non-relativistic theory. Course of Theoretical Physics, Vol. 3, Addison-Wesley Series in Advanced Physics, Pergamon Press Ltd., London-Paris, 1958, Translated from the Russian by J. B. Sykes and J. S. Bell.

[NvdS90] Henk Nijmeijer and Arjan van der Schaft, Nonlinear dynamical control systems, Springer-Verlag, New York, 1990.

[PW66] M. H. Protter and H. F. Weinberger, On the spectrum of general second order operators, Bull. Amer. Math. Soc. 72 (1966), 251-255.

[RBT00] Luc Rey-Bellet and Lawrence E. Thomas, Asymptotic behavior of thermal nonequilibrium steady states for a driven chain of anharmonic oscillators, Comm. Math. Phys. 215 (2000), no. 1, 1-24.

[RBT02] - Exponential convergence to non-equilibrium stationary states in classical statistical mechanics, Comm. Math. Phys. 225 (2002), no. 2, 305-329.

[SL77] Herbert Spohn and Joel L. Lebowitz, Stationary non-equilibrium states of infinite harmonic systems, Comm. Math. Phys. 54 (1977), no. 2, 97-120.

[SV72a] D. Stroock and S. R. S. Varadhan, On degenerate elliptic-parabolic operators of second order and their associated diffusions, Comm. Pure Appl. Math. 25 (1972), 651-713.

[SV72b] Daniel W. Stroock and S. R. S. Varadhan, On the support of diffusion processes with applications to the strong maximum principle, Proceedings of the Sixth Berkeley Symposium on Mathematical Statistics and Probability (Univ. California, Berkeley, Calif., 1970/1971), Vol. III: Probability theory (Berkeley, Calif.), Univ. California Press, 1972, pp. 333-359.

Jean-Philippe Aguilar

Centre de Physique Théorique (CPT)

CNRS, UMR 6207

Campus de Luminy, Case 907

13288 Marseille Cedex 9, France

E-mail address: aguilar@cpt.univ-mrs.fr

Nils Berglund

Université d'Orléans, Laboratoire MAPMO

CNRS, UMR 6628

Fédération Denis Poisson, FR 2964

Bâtiment de Mathématiques, B.P. 6759

45067 Orléans Cedex 2, France

E-mail address: nils.berglund@univ-orleans.fr 